\title{
Arbeiterbewegung von rechts? Motive und Grenzen einer imaginären Revolte
}

\author{
Klaus Dörre $\cdot$ Sophie Bose $\cdot$ John Lütten $\cdot$ Jakob Köster
}

Online publiziert: 22. Mai 2018

(C) Der/die Autor(en) 2018

Zusammenfassung Der Beitrag befasst sich mit völkisch-populistischen Orientierungen von betrieblich aktiven, gewerkschaftlich organisierten und teilweise in Betriebsräten aktiven Arbeitern. Anhand einer empirischen Tiefenbohrung wird gezeigt, wie sich im Alltagsbewusstsein der Befragten Protestmotive mit einer Ethnisierung der sozialen Frage verbinden. Der völkische Populismus kann als Bewegung Polanyi'schen Typs interpretiert werden, die sich aus dem Problemrohstoff des zeitgenössischen Postwachstumskapitalismus speist und sich als Demokratisierungsprojekt präsentiert. Imaginär bleibt die populistische Revolte, weil sie, letztendlich herrschaftskonform, auf eine Wiederherstellung von Verhältnissen zielt, die nicht wiederherstellbar sind. Unsere empirischen Befunde verhalten sich zu monokausalen Erklärungen sperrig, verweisen jedoch auf eine verdrängte Klassenproblematik. Weil es aussichtslos erscheint, als ungerecht empfundene Verteilungsverhältnisse grundlegend zu korrigieren, neigen Lohnabhängige spontan dazu, Auseinandersetzungen zwischen oben und unten in Konflikte zwischen innen und außen umzudefinieren. Die Tendenz zu exklusiver Solidarität wird vom organisierten Rechtspopulismus

K. Dörre $(\bowtie) \cdot$ J. Lütten · J. Köster

Institut für Soziologie, Friedrich-Schiller-Universität Jena, Carl-Zeiß-Straße 3, 07743 Jena,

Deutschland

E-Mail: klaus.doerre@uni-jena.de

J. Lütten

E-Mail: john.luetten@uni-jena.de

J. Köster

E-Mail: jakob.koester@uni-jena.de

S. Bose

DFG-Kolleg „Postwachstumsgesellschaften“, Friedrich-Schiller-Universität Jena,

Humboldtstr. 34, 07743 Jena, Deutschland

E-Mail: sophie.bose@uni-jena.de 
aufgegriffen und verstärkt - eine Herausforderung für demokratische Zivilgesellschaften und die Gewerkschaften.

Schlüsselwörter Arbeiter - Gewerkschaften - Ethnopluralismus · Exklusive Solidarität · Klassengesellschaft · Klassenpolitik · Postwachstumskapitalismus · Verteilungskonflikte $\cdot$ Rassismus $\cdot$ Rechtspopulismus $\cdot$ Rechtsextremismus

\section{A Labor Movement from the Right? Motives and limits of an imaginary revolt}

Abstract The article examines populist and völkisch orientations of unionized workers, some of them active members of workers' councils. It empirically shows how, in respondents' everyday consciousness, protest motifs are intermingled with an ethnicized view of the social question. Völkisch populism can be interpreted as a Polanyi-type movement that is motivated by problems generated by post-growth capitalism, presenting itself as a venture to give back power to the people. Its revolt remains an imaginary one, though, for, ultimately in accordance with existing power relations, it aims at reconstructing an irretrievable past. Our empirical results keep a distance from monocausal explanations, though call to mind issues of class that have long been neglected. As workers perceive the current distribution of wealth as unjust, yet don't believe in any possibility for change, they are spontaneously inclined to redefine existing top-down conflicts into inclusion-exclusion types. Professional right-wing populists take up and aggravate this tendency of exclusive solidarity and thereby pose a serious challenge for the unions as well as for democratic civil societies.

Keywords Workers · Unions · Ethnopluralism · Exclusive solidarity · Class society - Class politics · Post-growth capitalism - Distributional conflicts · Racism · Right-wing populism $\cdot$ Right-wing extremism

\section{Un mouvement ouvrier de droite? Motifs et limites d'une révolte imaginaire}

Résumé Cet article se penche sur les orientations populistes à caractère ethnonationaliste (völkisch) d'ouvriers engagés au sein de l'entreprise, organisés dans des syndicats et, pour certains, membres du comité d'entreprise. À l'aide d'une étude empirique approfondie, il est montré comment dans la conscience quotidienne des personnes interrogées les motifs de protestation se conjuguent à une ethnicisation de la question sociale. Le populisme ethno-nationaliste peut être interprété comme un mouvement au sens de Polanyi qui est alimenté par les problèmes générés par le capitalisme post-croissance contemporain et se présente comme un projet de démocratisation. Cependant, cette révolte populiste reste imaginaire en ceci qu'elle vise le rétablissement de conditions qui ne sauraient être rétablies, faisant ainsi finalement le jeu de la domination. Les résultats de cette étude empirique ne se prêtent pas aisément à des explications monocausales mais renvoient à une problématique de classe refoulée. Comme il paraît vain de corriger fondamentalement un régime de 
répartition ressenti comme injuste, les salariés tendent spontanément à redéfinir les conflits entre le haut et le bas en conflits entre l'intérieur et l'extérieur. Les organisations de la droite populiste s'emparent de cette tendance à la solidarité exclusive et la renforcent, posant ainsi un défi aux sociétés civiles démocratiques et aux syndicats.

Mots-clés Ouvriers · Syndicats · Pluralisme ethnique - Solidarité exclusive · Société de classes · Politique de classes - Capitalisme post-croissance Conflits de répartition $\cdot$ Racisme $\cdot$ Populisme droite $\cdot$ Extrême droite

\section{Einleitung: Aufstand der Mitte oder Arbeiterrevolte?}

„Für alle reicht es nicht“, titelt ein Band mit Texten Heiner Müllers. Kapitalismus, so die provokante Botschaft, beruhe stets auf dem Prinzip der Selektion (Müller 2017, S. 254 ff.; kritisch: Götze 2017). „Für alle reicht es nicht“ könnte auch der Leitsatz jener imaginären Revolte (Steil 1984; Weiß 2017) von rechts sein, die in vielen Ländern innerhalb wie außerhalb Europas die politische Agenda bestimmt. Während Heiner Müller dem Aussortieren ein entschlossenes ,alle oder keiner“ (Müller 2017, S. 79) entgegensetzt, radikalisiert die neue Rechte die alltägliche Erfahrung vieler Lohnabhängiger, dass es trotz steigenden Wohlstands nicht mehr für alle und alles reicht. Der völkische Populismus ${ }^{1}$ will Wohlstand, Arbeit und gutes Leben bevorzugt für éthnos, für ein homogenes Volk nur der Einheimischen, das durch Geburt, Blutsbande, Tradition und Kultur zusammengehalten wird. Damit hat er Erfolg - auch in der reichen Bundesrepublik. Was sich mit Pegida und deren Ablegern außerparlamentarisch andeutete und in regionalen Wahlerfolgen der Alternative für Deutschland (AfD) seine Fortsetzung fand, ist seit dem 25. September 2017 auf neuem Level etabliert. Die populistische Revolte besitzt nun eine parlamentarische Vertretung im Bundestag. Was bedeutet dieser Aufstieg einer neuen Rechten? Und vor allem: Wie ist er zu erklären?

An den Antworten scheiden sich in den Sozialwissenschaften die Geister. Pegida und AfD repräsentierten einen Bürgerprotest, der auf Repräsentationsdefizite im politischen System reagiere, eine überfällige Normalisierung des Parteiensystems darstelle und mit der „Funktionslogik pluralistischer Demokratie“ (Patzelt 2016, S. 80) zu befrieden sei, behaupten die einen. Es handele sich um einen Bruch in der politischen Kultur, ja um den „Saatboden für einen neuen Faschismus“ (Habermas 2016, S. 39), entgegnen andere. Wo manche einen kulturellen Konflikt ausmachen, der von den Mittelschichten ausgeht oder innerhalb der sozialen Mitte ausgetra-

\footnotetext{
1 Die Begriffe völkischer Nationalismus, völkische Rechte oder völkischer Populismus werden hier synonym und als Konkretisierung von Rechtspopulismus verwendet. Sie signalisieren, dass Arbeit am Begriff notwendig ist (Heitmeyer 2018). Rechtspopulismus und Rechtsextremismus werden kategorial im Zusammenhang mit dem heuristischen Rahmen der Untersuchung präzisiert (siehe die Ausführungen in Abschnitt 3.2).
} 
gen wird (Koppetsch 2017, S. 212; Lengfeld 2017), sprechen Kontrahentinnen ${ }^{2}$ im Anschluss an Didier Eribon (2016) oder Arlie Hochschild (2017a) von ,neuen Arbeiterparteien“ (Gertz 2017; differenzierter: Jörke und Nachtwey 2017; S. 174), die den Habenichtsen einen wirksamen Protest gegen das Establishment und dessen „progressiven Neoliberalismus“ (Fraser 2017) ermöglichten. Zwischen den Polen bewegt sich, wer neue Spaltungslinien ,,weniger in den ökonomischen als in der kulturell-identitären Sphäre“ verortet (Merkel 2017, S. 56), Spannungen zwischen „kulturellen Klassen“ aber klar benennt (Reckwitz 2017, S. 273 ff.).

Wie immer man die überwiegend auf Daten aus der Wahl- und Umfrageforschung beruhenden Diagnosen jedoch dreht und wendet - stets ergibt sich der gleiche Befund. Der völkische Nationalismus findet auch in Deutschland in allen Bevölkerungsgruppen Gehör. Bei Arbeitern, Gewerkschaftsmitgliedern und Erwerbslosen stößt er indes auf überdurchschnittliche Resonanz. Mit 12,6\% der Stimmen in den Bundestag eingezogen, votierten $19 \%$ der Arbeiterinnen und 15\% der Gewerkschaftsmitglieder (14\% West, $22 \%$ Ost, DGB 2017; Forschungsgruppe Wahlen 2017) für die populistische Formation. ${ }^{3}$ Die meisten AfD-Wähler haben die mittlere Reife oder den Hauptschulabschluss, nur 7\% der Akademiker wählen die AfD. Frauen sind im Elektorat der Partei deutlich unterrepräsentiert; dafür sind die Anteile in ländlichen und strukturschwachen Regionen besonders hoch (Forschungsgruppe Wahlen 2017). Betrachtet man anstelle des von taktischen Kalkülen beeinflussten Wahlverhaltens die aussagekräftigeren Parteipräferenzen, ergibt sich ein ähnliches Bild. Im Vergleich zu allen anderen Parteien weist die AfD die größte Einkommensspreizung, aber auch die höchsten Anteile an Arbeitern sowie abhängig Beschäftigten mit einfachen Arbeitstätigkeiten auf (Brenke und Kritikos 2017). Das Sozialprofil von Pegida wird ebenfalls von Arbeitern und Angestellten mit niedrigen bis mittleren Einkommen geprägt (Patzelt und Klose 2016; Geiges et al. 2015, S. 68). In Selbstdarstellungen präsentiert sich die Bewegung als Bündnis von „Mittelstand und Arbeiterklasse“ (Pegida 2015). Ähnlich agiert die AfD, wenn sie die „kleinen Leute“ als wichtige Zielgruppen ihrer Wahlkämpfe adressiert (Friedrich 2016, S. 231).

Am Zuspruch nicht zuletzt gewerkschaftlich organisierter Arbeiterinnen für den rechten Populismus setzt unser Beitrag an. Völkisch-populistische Orientierungen von Lohnabhängigen sind ein vielschichtiges Phänomen, das sich nicht auf das eine Motiv, die eine Ursache zurückführen lässt. Sie besitzen jedoch, so unsere These, ein empirisch identifizierbares Zentrum. Sofern realistische Alternativen fehlen, tendieren selbst Beschäftigte in gesicherten Verhältnissen dazu, den Kampf um Statuserhalt oder -verbesserung mit Hilfe von Ressentiments auszutragen. Präziser: In einem von vertikalen Ungleichheiten geprägten Postwachstumskapitalismus, der mobilisierungsfähige intellektuelle Überzeugungssysteme für solidarisches Handeln marginalisiert, machen sich Klassenverhältnisse und Verteilungskämpfe bevorzugt im Modus der Konkurrenz, über eine permanente Scheidung der Gewinner von den

\footnotetext{
2 Im Folgenden werden aus Gründen der Lesbarkeit anstelle der weiblichen und männlichen Berufs- bzw. Akteursbezeichnungen die femininen und maskulinen Formen im unregelmäßigen Wechsel verwendet, wobei alle Geschlechter mit eingeschlossen sind.

3 Die Überrepräsentanz von Arbeitern in den Gewerkschaften bedingt die überdurchschnittlich ausgeprägte Sympathie von Gewerkschaftsmitgliedern für die äußerste Rechte (Hilmer et al. 2017).
} 
Verlierern sowie mittels kollektiver Abwertungen und Ausgrenzung sozialer Großgruppen bemerkbar. Sie wirken sozioökonomisch wie kulturell selektiv. Spontan können sie eine exklusive Solidarität Lohnabhängiger hervorbringen, die sich nicht allein gegenüber dem Kapital, dem Management oder den Vorgesetzten, sondern auch von anders und unten abgrenzt. Entsprechende Weltsichten bieten sich für eine Radikalisierung durch die äußerste Rechte geradezu an. Die Begründung dieser These erfolgt auf der Grundlage eigener empirischer Erhebungen. Nachfolgend werden Forschungsstrategie und heuristischer Rahmen der Untersuchung vorgestellt (2 und 3), sodann rechtspopulistische Orientierungen von gewerkschaftlich aktiven Arbeiterinnen samt der Vorstellungen von Demokratie und Gesellschaft rekonstruiert (4 und 5). Abschließend gehen wir noch einmal auf die eingangs angerissene Kontroverse zu den Ursachen des Rechtspopulismus ein und beleuchten Konsequenzen für Zivilgesellschaft und Gewerkschaften (6).

\section{Tiefenbohrung im gewerkschaftlichen Arbeitermilieu}

Rechtspopulistische Orientierungen von Lohnabhängigen haben wir in mehreren Studien erforscht, deren aktuellste sich einer Schockerfahrung verdankt. Dass es in der Gesellschaft ein erhebliches Potenzial an menschenfeindlichen, rechtspopulistischen oder rechtsextremen Orientierungen gibt, ist seit langem bekannt (Heitmeyer 2017). Die Neigung gewerkschaftlich organisierter Arbeiter zu solchen Orientierungen ist ebenfalls kein neues Phänomen. Untersuchungen, die eine Verbreitung rechtsextremer oder -populistischer Einstellungen in den Gewerkschaften belegen (zusammenfassend: Bibouche et al. 2009), hatten bereits nach der Jahrtausendwende für Debatten gesorgt. Seither gilt als gesichert, dass radikal rechte Positionen unter aktiven Gewerkschafterinnen auf entschiedene Ablehnung stoßen und mit demokratischer Partizipation (Zeuner et al. 2007) wirksam zu bekämpfen sind. Diese Gewissheit kann, so unser irritierender Befund, nicht mehr uneingeschränkt gelten. Aktive Gewerkschafterinnen, die in ihren Betrieben für steigende Organisationsgrade sorgen, sind teilweise bereit, eigenständig die Busse zu beschaffen, mit denen sie zu Pegida-Demonstrationen fahren. In der Selbstwahrnehmung handelt es sich um einander ergänzende Facetten demokratischen Aufbegehrens - mit der Gewerkschaft in Betrieb und Unternehmen, in der Gesellschaft mit Pegida und der AfD. Auf die Frage, ob Pegida eine Demokratiebewegung sei, antwortet ein sympathisierender Betriebsrat: „Ich denke schon“. Theoretisch könne die Bewegung ,,jeden ansprechen“; zwar schwebe ,ein Nazi-Schatten“ über ihr, doch sie thematisiere, was eigentlich ,,jeden Normalen betrifft, der in Lohn und Brot steht“" (Pro1).

\subsection{Empirische Basis, Methodik}

Pegida und die AfD als Demokratiebewegung normaler Lohnabhängiger - das ist der Tenor von Selbstdarstellungen radikal rechts orientierter Arbeiter, die wir im Rahmen eines laufenden Forschungsvorhabens zum „Gesellschaftsbild des Prekariats“ erhoben haben. Unsere Primärerhebung $(\mathrm{n}=66)$, die als Teilprojekt eine Tiefenbohrung $(\mathrm{n}=16)$ in sächsischen Regionen beinhaltet, aktualisiert eine Basis- 
studie zu Prekarität und Rechtspopulismus aus den Jahren 2003-2006. Empirische Grundlage dieser Basisstudie sind themenzentrierte Interviews mit Arbeitern und Angestellten ( $\mathrm{n}=100)$, darunter viele prekär Beschäftigte und Erwerbslose, Expertengespräche mit Führungskräften, Betriebsratsmitgliedern und Gewerkschafterinnen $(n=36)$ sowie eine Gruppenbefragung von Leiharbeitskräften, die wir nun mit einer neu zusammengesetzten Forschungsgruppe reinterpretieren. Die Basisstudie prüfte eine Arbeitshypothese Robert Castels, der zufolge sich die nachfordistischen Arbeitsgesellschaften in drei Zonen spalten - eine schrumpfende Zone der Integration mit noch immer gesicherten Arbeitsverhältnissen, eine expandierende Zone der Prekarität mit unsicherer, schlecht entlohnter, wenig anerkannter Beschäftigung sowie eine Zone der Entkoppelung mit all jenen, die kaum noch eine Chance auf ein reguläres Erwerbsverhältnis besitzen (Castel 2000, S. 360f.). Anhand dieser Heuristik rekonstruierten wir typische Verarbeitungsformen sozialer Unsicherheit, die dann als Hintergrundfolie für eine Identifikation rechtspopulistischer Orientierungen dienten (Brinkmann et al. 2006; Dörre 2009). Eine vertiefende Analyse von Gesprächssequenzen mit fremdenfeindlichen, autoritär-antidemokratischen oder antiegalitären Deutungsmustern förderte in einem induktiven Verfahren bei mehr als einem Drittel der Befragten Weltsichten mit Nähe zum Rechtspopulismus zutage (Dörre 2006, S. 157; Dörre et al. 2006). In unterschiedlichen Ausprägungen fanden sich solche Orientierungen in allen Zonen der Arbeitsgesellschaft. Bei Erwerbslosen und Facharbeiterinnen waren sie ebenso präsent wie bei Bankangestellten in leitender Funktion. Überwiegend hatten sich diese Orientierungen aber nicht parteipolitisch verfestigt, sondern machten sich als rechtspopulistische Unterströmung in Gewerkschaften und demokratischen Parteien bemerkbar.

Wie die Basisstudie, so ordnet auch die Primärerhebung aus den Jahren 2017/18 befragte Lohnabhängige aus Ost und West anhand ihres Beschäftigungsstatus den Castel'schen Zonen zu. Allerdings handelt es sich um ein ,unechtes Panel“, denn die befragten Personen sind nicht mehr die der Basisstudie. Wir präsentieren eine zeitversetzte Neubefragung, die gleichwohl methodisch reflektierte Aussagen im Zeitverlauf ermöglicht ${ }^{4}$. Auch die Zuordnung Befragter zum Rechtspopulismus hat sich geändert. Um der Bewegungsförmigkeit und parteipolitischen Verselbständigung des Rechtspopulismus Rechnung zu tragen, setzt die Primärerhebung das offene Bekenntnis zu entsprechenden Formationen als Kriterium voraus. Daran gemessen sympathisieren von 66 Befragten insgesamt neun (sieben Männer, zwei Frauen) offen mit Pegida, der AfD oder weiter rechts stehenden Organisationen (Reichsbürger, NPD, Nazi-Skins). Hätten wir als Kriterien diejenigen der Basisstudie gewählt, wäre die Fallzahl von Befragten mit Affinitäten zum Rechtspopulismus größer, denn Ressentiments gegen Fremde finden sich auch bei Lohnabhängigen, die sich politisch eindeutig links verorten.

\footnotetext{
4 „Unechte Panels“ sind in der Arbeitssoziologie durchaus üblich; als prominentes Beispiel siehe Kern und Schumann (1984).
} 
Um die empirische Basis unserer Untersuchung noch zu verbreitern, können wir mittels Daten- und Forscherinnen-Triangulation (Denzin 1989; Flick 2008) weitere Erhebungen aus dem Zeitraum von 2006 bis 2016 in die Analyse einbeziehen. ${ }^{5}$ Triangulation meint hier neben der Sichtung des Materials durch mehrere Forschungsgruppen vor allem die Integration unterschiedlicher qualitativer Datensätze in einen übergreifenden, theoriegeleiteten heuristischen Rahmen. Die eigentliche soziologische Tiefenbohrung, die uns möglichst nah an das Alltagsbewusstsein rechter Arbeiterinnen heranbringen sollte, beruht auf 16 Interviews der Primärerhebung. Unter Tiefenbohrung verstehen wir eine Forschung, die sich Schritt für Schritt an ihren Gegenstand herantastet. Rechte Arbeiter sind, anders als in der Basisstudie, keine Zufallsfunde. Wir haben diese Personen im Rahmen der Tiefenbohrung bewusst gesucht und befragt (Hochschild 2017b).

\subsection{Forschung als öffentliche Soziologie}

Um die Gespräche führen zu können, war ein aufwendiges Verfahren nötig. Zunächst identifizierten wir innerhalb eines breiter angelegten Samples acht betriebliche Fallbeispiele in Ost und West, in denen radikal rechte Positionierungen von Interessenvertretern oder Beschäftigten auffällig geworden waren. Im nächsten Schritt wählten wir eine sächsische Region aus, die durch eine außergewöhnlich hohe Zustimmung der Bevölkerung zu Pegida und AfD aufgefallen war. Hauptamtliche Gewerkschafterinnen halfen uns bei der Auswahl geeigneter Gesprächspartner. Die Interviews fanden an mehreren Tagen in den Räumlichkeiten der Gewerkschaft statt. Alle Interviewpartner wurden einzeln befragt. Thematisch lag der Schwerpunkt beim Verhältnis zu Pegida/der AfD, dem Gewerkschaftsverständnis sowie der subjektiven Sicht auf Arbeit, Betrieb, Gesellschaft und Demokratie. Die Tiefenbohrung umfasst Interviews mit hauptamtlichen Gewerkschaftssekretärinnen (codiert als Sek), radikal rechten Betriebsräten und Gewerkschaftern (codiert als Pro); gewerkschaftlich organisierten Beschäftigten mit Anti-Pegida/AfD-Haltung (codiert als Ant) sowie Jugendvertreterinnen (codiert als JAV). Hauptamtliche Gewerkschafter wurden als Experten befragt (Bogner et al. 2005; Gläser und Laudel 2004), bei den betrieblich Aktiven kamen problemzentrierte Interviews (Kaufmann 1999, S. 24; Witzel 2000) zum Einsatz, die inhaltsanalytisch ausgewertet wurden (Kelle und Kluge 2010, S. 43 f.; Gläser und Laudel 2004, S. 191 f.; Mayring 2003).

Sowohl die Experten- als auch die problemzentrierten Interviews enthalten längere Passagen des Stehgreiferzählens. Mit Hilfe theoriegeleiteter Codierung haben wir solche Interviewpassagen in reflektierender Interpretation (Bohnsack 1993, S. 132-138) nach Widersprüchen, Ungereimtheiten und sprachlichem Duktus untersucht, um so deren latenten Sinn zu erfassen. Die befragten Pro-Pegida-Arbeiter sollten ein möglichst realistisches Gesamtbild repräsentieren. Mit Hilfe eines theoretical sampling (Glaser und Strauss 1998, S. 51 ff.) haben wir drei Fälle für eine intensive Auswertung ausgewählt, die jeweils für Abstufungen einer völkisch-populistischen

\footnotetext{
5 Einbezogen werden Studien zu gewerkschaftlicher Erneuerung in Ost und West (Dörre et al. 2017), eine Mehrfachbefragung von Leistungsbeziehern des ALGII (Dörre et al. 2013a) sowie mehrere Datensätze aus einem Forschungsvorhaben zum „Gesellschaftsbild von LohnarbeiterInnen“ (Dörre et al. 2013b).
} 
Grundhaltung stehen. Wir rekonstruieren Orientierungen des Protestwählers, des gefestigten Pegida/AfD-Anhängers und des überzeugten Rechtsextremen (codiert als Pro 1, 2, 3). Andere Befragte mit radikal rechten Orientierungen werden punktuell und zur Differenzierung von Aussagen in die Auswertung einbezogen. Was für eine qualitative Erhebung konventionell erscheinen mag, entpuppte sich selbst für forschungserfahrene Wissenschaftlerinnen als Grenzsituation. In allen Fällen gelang es, eine offene Gesprächsatmosphäre herzustellen. Dennoch oder gerade deshalb erwiesen sich die Interviews mit den Pro-Pegida/AfD-Befragten als Herausforderung. Wie soll man beispielsweise mit einem Gewerkschafter umgehen, der dafür plädiert, wegen der Fluchtmigration das Lager Buchenwald wieder zu eröffnen? Wir haben uns bemüht, die Fassung zu wahren, das Interview fortgesetzt und uns am Schluss höflich per Handschlag verabschiedet.

Angemerkt sei, dass wir das Feld nur erschließen konnten, weil wir praktizierten, was im Anschluss an Michael Burawoy als organische „Public Sociology“ bezeichnet werden kann (Burawoy 2015; Aulenbacher et al. 2017). Öffentliche Soziologie ist organisch, wenn sie ,in enger Verbindung mit einer sichtbaren, dichten, aktiven, lokalen Öffentlichkeit arbeitet, die oft eine Gegenöffentlichkeit ist" (Burawoy 2015, S. 57). Im konkreten Fall kooperierten wir mit hauptamtlichen Gewerkschaftssekretärinnen, denen es vor allem darum ging, innerhalb der eigenen Organisation Aufmerksamkeit für die Rechtspopulismus-Problematik zu erzeugen. Eine klare Positionierung des IG Metall-Vorsitzenden (,Wer hetzt, fliegt!“) hatte in der Geschäftsstelle zum schriftlich begründeten Austritt von ca. 30 Mitgliedern geführt; die Gesamtzahl ähnlich motivierter Austritte wird von verantwortlichen Sekretären auf bis zu 300 geschätzt (zu ähnlichen Fällen im Westen: Sauer et al. 2018, S. 189 ff.). Trotz des Aderlasses gibt es im Kreis der aktiven Gewerkschafter noch immer erhebliche Sympathien für Pegida und AfD. Hauptamtlichen Sekretärinnen, die sich allesamt gegen den völkischen Populismus positionieren, suchen nach einer geeigneten Strategie für eine Auseinandersetzung mit langem Atem. Neben dem Interesse, innergewerkschaftlich ein Problembewusstsein zu schaffen, ist es ihnen wichtig, ein wissenschaftliches Feedback zu den vor Ort praktizierten unterschiedlichen Linien im Umgang mit rechten Mitgliedern zu erhalten.

Unsere Studie sollte diese Erwartungshaltung bedienen. Nach intensiver Vorbereitung wurden erste Untersuchungsergebnisse zum Thema einer Delegiertenversammlung der regionalen Gewerkschaftsgliederung. Die Präsentation war ein Härtetest für unsere Forschungen und sie führte zu einem überraschenden Ergebnis. Erstmals äußerten sich anwesende Delegierte öffentlich zu Pegida und der AfD, sprachen über Gewerkschaftsaustritte und problematisierten im Beisein eines geschäftsführenden Vorstandsmitglieds der IG Metall die „Klare-Kante-Politik“. Für einen Augenblick war es gelungen, zuvor Verborgenes ansatzweise diskutierbar zu machen. Was wir zutage gefördert haben, ist nicht nur für lokale Gewerkschaftsgliederungen schmerzlich. Sympathien für die äußerste Rechte mögen regional besonders ausgeprägt sein, es handelt sich aber beileibe nicht um einen Einzelfall. In insgesamt 18 Gewerkschaftsgliederungen, in denen wir die Ergebnisse unserer Tiefenbohrung präsentierten (festgehalten in Memos), fielen die Reaktionen ähnlich aus. Man hielt die Problematik für relevant und brisant, plädierte mehrheitlich aber für interne Diskussionen, weil „uns sonst der Laden um die Ohren fliegen könnte“ (Memo 2). 


\section{Der heuristische Rahmen}

Bevor wir genauer beleuchten, was sich hinter solchen Äußerungen verbirgt, empfiehlt es sich, den übergreifenden heuristischen Rahmen der Untersuchung vorzustellen. Inhaltlich zentral sind Zusammenhänge zwischen sozialer Frage und Rechtspopulismus, die sich jedoch während der Zeit, die seit der Basisstudie vergangen ist, erheblich verändert haben. Präziser: Die Bewährungsproben, also das Kräftemessen und die Wertigkeitsprüfungen, in denen über die Akzeptanz sozialer Ungleichheiten entschieden wird (Boltanski und Chiapello 2003), haben sich in ihren Formaten und ihrer Gewichtung erheblich gewandelt.

\subsection{Von der Prekarität zur Bewährungsprobe des Lohns}

Unsere Basisstudie hatte die soziale Frage noch mit der Ausbreitung unsicherer Arbeits- und Lebensverhältnisse gleichgesetzt (Brinkmann et al. 2006). In der Bewährungsprobe der Prekarität entschied sich für erhebliche Teile der Lohnabhängigen, ob der Sprung in sichere Beschäftigung und damit in die Gesellschaft respektierter Bürgerinnen gelingen konnte. Prekarität, so der noch immer gültige Befund aus der Basisstudie, bezeichnet keine stabile soziale Lage, sondern ein Macht- und Kontrollsystem, das auch gesichert Beschäftigte diszipliniert und so zur Herausbildung gefügiger Arbeitskräfte beiträgt (Dörre 2009, S. 54 ff.; Bourdieu 1998, S. 97 f.). Im Zeitverlauf hat diese disziplinierende Wirkung jedoch nachgelassen. Dagegen haben Bewährungsproben, in denen es um die Verteilung des erzeugten Mehrprodukts, um Löhne und Arbeitszeiten geht, auch subjektiv wieder an Bedeutung gewonnen. Das hängt wesentlich mit Veränderungen am Arbeitsmarkt zusammen. Die offizielle Arbeitslosenquote, die 2005 mit 11,7\% ihren Höchstwert erreicht hatte, ist von 9,5 auf unter $6 \%$ gesunken. Parallel dazu hat die Zahl der Erwerbstätigen zwischen 1991 und 2014 um ca. 3,7 Mio. Beschäftigte zugenommen und mit mehr als 44,7 Mio. Erwerbspersonen (2014) Rekordniveau erreicht (Destatis 2016, S. 126).

Prekarität ist damit aber keineswegs verschwunden, sondern im Gegenteil zu einer normalen Organisationsform von Arbeit und Leben avanciert. Trotz günstiger konjunktureller und demografischer Entwicklung sind atypische, unsichere und niedrig entlohnte Beschäftigungsformen seit 2010 nur leicht zurückgegangen, Leiharbeit und Werkverträge haben sogar zugelegt. Während der Niedriglohnsektor kontinuierlich 22 bis $24 \%$, im Osten gar bis zu $37 \%$ der abhängig Beschäftigten umfasst, lag das ungenutzte Arbeitskräftepotenzial 2014 noch immer bei ca. 6 Mio. Personen (2,1 Mio. Erwerbslose, 2,9 Mio. Unterbeschäftigte, 1,0 Mio. stille Reserve). Auch das Volumen bezahlter Erwerbsarbeitsstunden bewegte sich 2016 noch immer unter dem Niveau von 1991. Leistete ein Erwerbstätiger 1991 jährlich 1554 Arbeitsstunden, so waren es 2014 nur 1366, das entspricht einem Rückgang um $12 \%$ bei allerdings stark polarisierten Arbeitszeiten (Destatis 2016, S. 127). Rechnet man die gewünschten Arbeitszeiten unterbeschäftigter Personen hinzu (Fischer et al. 2015), wäre die Erwerbslosigkeit mindestens doppelt so hoch wie offiziell ausgewiesen. Das deutsche Jobwunder beruht somit wesentlich darauf, dass Erwerbslosigkeit mittels Ausdehnung unsicherer, schlechter entlohnter und wenig anerkannter Arbeitsverhältnisse zum Verschwinden gebracht wird (Castel 2011, S. 136). 
Fügt sich die Herausbildung einer prekären Vollerwerbsgesellschaft noch gut in das Raster des Castel'schen Zonenmodells, trifft das auf die zunehmende Vermögens- und Einkommensungleichheit so nicht zu. Selbst liberale Ökonomen bezeichnen die Bundesrepublik inzwischen als ,eines der ungleichsten Länder in der industrialisierten Welt“ (Fratzscher 2016, S. 9, 43 ff.). Das Vermögen der 45 reichsten Haushalte entspricht, nach den um zusätzliches Material erweiterten Daten der Europäischen Zentralbank, in etwa dem der ärmeren Bevölkerungshälfte. 0,1\% besitzen einen Anteil von 17,4\% des auf 9,5 Billionen Euro geschätzten Gesamtvermögens; $50 \%$ der Bevölkerung verfügen hingegen nur über einen Anteil von 2,3\% (Bach et al. 2018). Bei den Einkommen zeichnen sich seit der Wiedervereinigung ebenfalls Konzentration und Polarisierung ab. Legt man die Markteinkommen zugrunde, verdient die ärmere Hälfte der Bevölkerung nur noch $17 \%$ des Volkseinkommens (1960: 30\%, Bartels 2018, S. 1). Wie in allen Industrieländern ist die Lohnquote (Anteil der Löhne am BIP) auch in Deutschland seit den 1980er Jahren kontinuierlich gesunken, um erst in der jüngeren Vergangenheit wieder leicht anzuziehen (IWF 2017). Während qualifizierte, gut organisierte Stammbeschäftigte in der Exportindustrie ihre Reallöhne halten oder steigern konnten (Hauptmann und Schmerer 2012, S. 3), mussten insbesondere Frauen im Dienstleistungssektor überdurchschnittliche Einbußen hinnehmen. Etwa die Hälfte der Lohnabhängigen verdiente 2015 real weniger als noch vor 15 Jahren (Fratzscher 2016, S. 64).

Die Polarisierungstendenz bei Löhnen und Einkommen macht auch vor den Organisationsbereichen der Industriegewerkschaften nicht halt. Zwar konnte die IG Metall für ihre Facharbeiterinnen seit der Jahrtausendwende einen Lohnzuwachs von mehr als $50 \%$ durchsetzen, doch profitierten in erster Linie die Stammbelegschaften großer und mittlerer Unternehmen. Schon in der Zulieferindustrie und hier besonders im Osten bietet sich ein anderes Bild. Wie im gesamten Osten hatte die IG Metall auch in den Untersuchungsregionen der Tiefenbohrung Stufentarifverträge mit der Aussicht verbunden, ,in zehn Jahren habt ihr den gleichen Tarifvertrag wie in West, die 35-Stunden-Woche und eins zu eins die Lohntabelle“ (Sek2). Dieses Versprechen konnte für den Großteil der betreuten Betriebe nicht eingelöst werden. Nur in besonders gut organisierten Unternehmen ist die Angleichung halbwegs gelungen, in weiten Bereichen von Metall, Elektro, Textil und Bekleidung bestehen „abweichende Regelungen“: „Wir haben hier die 38-, wenn nicht die 40-StundenWoche, wir haben immer zwischen 10 und $25 \%$ unterhalb der Lohntabelle West“" (Sek2).

Ungleichheit macht sich somit nicht allein in Verteilungsrelationen zwischen Kapital und Arbeit bemerkbar, sie fragmentiert innerhalb der lohnabhängigen Klassen. Im Osten sorgt dies besonders unter jüngeren Arbeitern für Unmut. Lohnverzicht zugunsten von Beschäftigungssicherheit wird nicht mehr ohne Weiteres akzeptiert: „Am liebsten brennen wir die Hütte ab, was geht mich der Laden an? Geht der pleite, dann geht der pleite. Geh ich zum nächsten“, bringt ein befragter Gewerkschafter (Sek8, Dörre et al. 2017, S. 113) eine in jüngeren Kohorten verbreitete Stimmung auf den Punkt. Selbst bei Hochkonjunktur reichen Unmut und gewerkschaftliche Kampfkraft aber nicht aus, um Gerechtigkeitslücken zu schließen. Wir bezeichnen dieses Missverhältnis als Tendenz zu einer demobilisierten Klassengesellschaft. Vertikale, überwiegend klassenspezifische Ungleichheiten prägen sich aus. Zugleich 
sind gewerkschaftliche und politische Organisationen, die auf der Konfliktachse von Kapital und Arbeit agieren, während der gesamten Nachkriegsgeschichte nie so schwach gewesen wie in der Gegenwart. So ist der gewerkschaftliche Organisationsgrad in der Bundesrepublik auf etwa 18\% gesunken. Das mag dazu beitragen, dass die Bewährungsproben des Lohns und die mit ihr verkoppelten Problematiken - Leistungsdruck, mangelnde Zeitsouveränität und autoritäre Firmenregimes gesellschaftlich keine adäquate Öffentlichkeit finden.

Die Klassenvergessenheit öffentlicher Diskurse schlägt sich auch in der Populismusdebatte nieder. Von Gerechtigkeitsproblemen getrieben, wird aus Sicht vieler sozialwissenschaftlicher Interpretinnen nur, wer arm, lange erwerbslos oder prekär beschäftigt ist. Alle, auf die dergleichen nicht zutrifft, werden der sozialen Mitte zugerechnet und haben, so der analytische Kurzschluss, andere als Gerechtigkeitsprobleme (IW 2017; Lengfeld 2017). Auf diese Weise verstellt der „Mythos Mitte“ (Kadritzke 2017) den Blick dafür, dass es sich auch bei gut verdienenden Fachkräften um Lohnabhängige handelt. Selbst Ingenieure in der verarbeitenden Industrie entsprechen in ihrer sozialen Positionierung eher jenen akademisch gebildeten Arbeitskräften, die vor Jahrzehnten als „,neue Arbeiterklasse“ (Touraine 1964; Mallet 1964; 1965; Hörning 1971; Deppe 1971) von sich reden machten. Dass ordentlich verdienende Arbeiter sich deutlich von den Mittelklassen abheben, stand in solchen Analysen außer Frage; teilweise wurde den qualifizierten „,neuen Arbeitern“ "gar eine besondere Militanz attestiert (Hamilton 1968; Goldthorpe et al. 1967). Heute sind solch anspruchsvolle Klassenanalysen nahezu vergessen. Die öffentliche De-Thematisierung von Klassen- und Ausbeutungsverhältnissen ändert aber nichts daran, dass all jene sich „kollektiv im Stich gelassen fühlen, die mit den Verhältnissen hinter diesen Wörtern objektiv zu tun haben“ (Eribon 2016, S. 122). Ungeachtet fehlender Öffentlichkeiten erzeugen soziale Spaltungen einen Problemrohstoff, der sich in unterschiedliche Richtungen politisieren lässt. Der Sozialpopulismus, den Pegida und die AfD praktizieren, ist ein Versuch, den Bewährungsproben um Lohn und Arbeitsbedingungen ein ethnisch-nationalistisches Format zu geben.

\subsection{Rechtspopulismus und Ethnopluralismus}

Rechtspopulismus ist ein schillernder Begriff, mit dem wir in der Basisstudie über seinerzeit akzeptierte Definitionen hinaus ${ }^{6}$ Formationen bezeichneten, die danach strebten, Elemente politischer Philosophien mit organisierten politischen Praktiken und habitualisierten Haltungen, Handlungs- und Deutungsschemata zu einem ,histo-

\footnotetext{
${ }^{6}$ Nach damals gängigen Definitionsversuchen bezeichnete Rechtspopulismus politische Formationen, die sich vom tradierten Rechtsextremismus abgrenzten und ein „Scharnier“ zwischen Nationalkonservatismus und organisiertem Rechtsextremismus bildeten. Laut Birsl und Lösche (2001) zeichnete sich der neue Rechtspopulismus durch fünf Merkmale aus: ethnisch oder nationalistisch begründeter Kollektivismus, der sich mit aggressiver Fremdenfeindlichkeit paart; gesellschaftliche Ordnungsvorstellungen, die soziale Ungleichheit naturalisieren; obrigkeitsstaatliches Denken und autoritäre Wertorientierungen; die Ablehnung von Parteiendemokratie, Meinungsfreiheit und Pluralismus sowie eine Neigung zu verschwörungstheoretischen Deutungen von Geschichte und Politik. Vom Rechtsextremismus unterschied sich der neue Rechtspopulismus vor allem durch seine Ablehnung gewaltsamer Systemveränderung. Wie sich zeigen wird, lässt sich dieses Kriterium inzwischen nur noch schwer anwenden.
} 
rischen Block“ zu verschmelzen. Nach diesem an Antonio Gramsci (1991 ff., S. 490, 1490) angelehnten Ideologie-Verständnis dürfen rechtspopulistische Orientierungen von Lohnabhängigen keinesfalls auf falsches Bewusstsein reduziert werden. Wichtiger ist ihr bon sense, der rationale Kern, den das Alltagsbewusstsein enthält (Hall 1989, S. 56-91). Als populistisch können entsprechende Ideologeme gelten, weil sie den Problemrohstoff, den die Gesellschaft erzeugt, in einem binären Schema bearbeiten. Dem korrupten Establishment wird ein unverdorbenes Volk gegenübergestellt, dessen Wille im Zweifelsfall mit dem der Populisten übereinstimmt (Priester 2012; Müller 2016). Völkisch und rechts wird der neue Populismus infolge der Verwendung eines ethnopluralistischen Ideengebäudes, das ein Grundrecht auf Verschiedenheit aller Menschen und Kulturen an die Stelle früherer Vorstellungen von nationaler oder rassischer Überlegenheit setzt (Decker 2004; Loch und Heitmeyer 2001). Bei oberflächlicher Betrachtung ein zeitgemäßes Konzept kultureller Autonomie, können mit Hilfe dieses Ideengebäudes wahlweise die Kolonisierung des globalen Südens, Bedrohungen durch den Amerikanismus, die Unterdrückung von Regionalismen in Europa oder eine Einwanderung in die Sozialsysteme attackiert werden. All das geschieht im Namen einer gleichsam naturalisierten, homogen Nationalkultur. Folgt man diesem „Rassismus ohne Rassebegriff“ (Taguieff 1991, S. 221 ff.; Balibar 1993, S. 148), sind nur unvermischte Kulturen stark, der melting pot kann hingegen nicht funktionieren. Deshalb sollen alle Menschen ihre Kultur leben können - und zwar am besten dort, wo die jeweilige Kultur ursprünglich beheimatet ist. Unversehens verwandelt sich so ein attraktiv klingendes Plädoyer für kulturelle Autonomie in eine Rechtfertigung von Abschottung, die, würde sie konsequent angewendet, eine Welt voller Apartheidsstaaten hervorbringen müsste.

Am ethnopluralistischen Ideengebäude als solchem hat sich seit der Basiserhebung wenig geändert. Auf der Ebene organisierter, medial vermittelter Politik und deren alltäglicher Akzeptanz ist der Wandel allerdings gravierend. Sarrazin- und Unterschichten-Debatte haben maßgeblich zu einer Popularisierung ethnopluralistischen Gedankenguts beitragen. Die Fähigkeit zu außerparlamentarischer Mobilisierung (Pegida und Ableger) sowie die parteipolitische Verselbständigung und parlamentarische Etablierung des Rechtspopulismus in Gestalt der AfD (Häusler 2016) sind ebenfalls neu. Als marktradikale Partei gegründet, entwickelt sich die AfD seither ähnlich wie ihre europäischen Vorbilder mehr und mehr zu einer völkischsozialpopulistischen Formation (Bieling 2017). Intern ist dieser Kurs allerdings umstritten. Während Nationalkonservative in der bürgerlichen Mitte die ,eigentlich revolutionäre Klasse“ sehen, deren Endzweck ,nicht die klassenlose Gesellschaft, sondern die Wiederherstellung der sozialen Marktwirtschaft“ (Jongen 2014) sei, intoniert der national-soziale Flügel eine radikalere Melodie. Die ,,soziale Frage der Gegenwart“ bestehe nicht primär in der „Verteilung des Volksvermögens von oben nach unten“, die ,neue deutsche soziale Frage des 21. Jahrhunderts“ sei ,die Frage nach der Verteilung des Volksvermögens von innen nach außen“ (Björn Höcke, zit. nach Paulus 2017). Setzt die liberal-konservative Strömung auf einen Volkskapitalismus, der Mittelstand und Arbeiterschaft versöhnen soll, hält der national-soziale Flügel den DGB-Mitgliedsorganisationen vor, den „Gegnerbezug zu den Arbeitgebern“ nicht glaubhaft zu praktizieren, weil die Gewerkschaftsspitzen selbst „oben“ 
seien und die Gliederungen eher gefügigen ,Staatsgewerkschaften“ ähnelten (zit. n. Fiedler 2018).

Bei ihren Versuchen, Marktradikalismus und soziale Gerechtigkeit auszubalancieren, erweisen sich Repräsentantinnen des organisierten Rechtspopulismus, gleich welcher Strömung sie angehören, als „Pioniere der Ambivalenz“ (Decker 2004, S. 30; Kann 1983, S. 371). Aus ihrer Sicht ist es kein Widerspruch, die Interessen heimischer Arbeiter und ihrer Familien zu betonen, um im gleichen Atemzug massive Steuersenkungen für Unternehmen zu fordern. Nur als Meister der Ambivalenz können sich Rechtspopulisten des Problemrohstoffs der sozialen Frage bemächtigen. Neu ist nicht die sozialpopulistische Botschaft als solche, wohl aber die Härte, Radikalität und Kompromisslosigkeit, mit der sie verkündet wird. In der Freund-FeindTerminologie des nationalkonservativen Staatsrechtlers Carl Schmitt wird die anhaltende Fluchtmigration als „Landnahme“ unzivilisierter Eindringlinge bezeichnet und zum Ausnahmezustand mit Bürgerkriegspotenzial hochstilisiert, weil sie den Wohlstand und die Kultur der deutschen Bevölkerung bedrohe (Kellershohn 2016). Exakt dies, die Verklammerung von Verteilungskämpfen und Migration, bindet von uns befragte Gewerkschafterinnen an den rechtspopulistischen Block. Für die Befragten macht es Sinn, AfD zu wählen, weil sie als „einzige Partei“ das Flüchtlingsthema „kritisch beäugt“ (Pro2). Wahlentscheidungen erfolgen als Protest oder aus Überzeugung, versehen mit dem Hinweis, man könne von einer jungen Partei kein Programm verlangen, das „100\% passend ist“ (Pro1). Die Flüchtlingskrise bildete jenen ,,populistischen Moment“ (Goodwyn 1978), der unsere Befragten dazu motivierte, für zumindest latent bereits vorhandene Orientierungen einen neuen parteipolitischen Adressaten zu suchen.

\section{Alltagsbewusstsein und rechtspopulistische Orientierungen}

Halten wir fest: Trotz Hochkonjunktur und sinkender Arbeitslosigkeit sehen sich Bevölkerungsmehrheiten mit der Herausbildung eines Postwachstumskapitalismus (Galbraith 2016, Dörre 2017) konfrontiert, für den die Erfahrung konstitutiv ist, dass es trotz - freilich relativ niedriger - Wachstumsraten nicht mehr für alle und alles reicht. Diese Schlüsselerfahrung prägt das Alltagsbewusstsein und die gesellschaftlichen Bewährungsproben. Allerdings wirken weder Prekarität noch klassenspezifische Ungleichheiten aus sich heraus in eine bestimmte politische Richtung. Das spontane Wissen der Beherrschten ,hat keine stabile Bedeutung oder politische Bindung“ (Eribon 2016, S. 142). Die Produktionsmodi politischer Meinungen (Klassenethos, explizite politische Prinzipien, Urteile zweiten Grades; Bourdieu 1988, S. 655-659) wirken relativ unabhängig von sozialen Lagen. Sie erzeugen Gesellschaftsbilder - Weltsichten oder Alltagsphilosophien - die zwischen der ,kleinen Welt" des eigenen Erfahrungshorizonts in Betrieb, Familie und Freundeskreis sowie der ,großen Welt“" politischer Konflikte und historischer Ereignisse vermitteln (Popitz et al. 1957, S. 163 ff.). Lebensgeschichtlich angeeignete Weltsichten schließen einfache Erklärungen nach dem Muster „Je größer der Problemdruck, desto wahrscheinlicher die Aufgeschlossenheit für rechtspopulistische Orientierungen“ aus. Es sind je besondere, empirisch aufzuklärende Umstände, die dazu führen, dass sich 
das Aufbegehren gegen Unsicherheit und Ungleichheit mit völkisch-nationalistischen Weltsichten verbindet.

\subsection{Dichotomie mit Zusatz: nicht „ganz unten“"}

Betrachten wir zunächst eine Grundhaltung, die sich bei vielen aktuell befragten Arbeiterinnen findet, gleich ob sie sich nun politisch links, rechts oder in der Mitte verorten. Ungeachtet aller sonstigen Unterschiede stoßen wir auf ein Gesellschaftsbild, das strikt zwischen oben und unten unterscheidet. „Meine Eltern sind beide Arbeiter. Ich habe kein Abi gemacht, kein Studium angefangen, habe halt meinen Realschulabschluss und meine Ausbildung gemacht und arbeite jetzt. Und ich bin mir relativ sicher, dass es dabei bleiben wird. Ich würde mich schon zur mittleren Mittelschicht zählen, aber dabei wird es einfach bleiben. Diese Spalte zwischen Mittelschicht [...] und der Oberschicht, die ist halt einfach riesengroß. Und ich werde diese Spalte niemals überspringen können [...], kann ich machen, was ich will. Und so geht es einfach sehr, sehr vielen“ (JAV1), erklärt eine zwanzigjährige Arbeiterin im Interview.

Treffend beschreibt die Befragte eine im Sample verbreitete Weltsicht. Die Mehrzahl der Arbeiterinnen aus der aktuellen Erhebung befindet sich am Beschäftigungsstatus gemessen in der Zone der Integration. Mit einem festen Job und einem gesicherten Einkommen glauben sie, alles erreicht zu haben, was sie erreichen können. Sie sind weder arm noch prekär und rechnen sich trotz teilweise bescheidener Löhne der „mittleren Mittelschicht“ zu. Bei Abschlüssen auf Realschulniveau ist der reproduktive Zirkel vorgezeichnet. Mit großer Wahrscheinlichkeit landet man bestenfalls dort, wo auch die Eltern positioniert sind. Ein Aufstieg durch Bildung ist unrealistisch. Wenn überhaupt, so wird es in Zukunft um graduelle Verbesserungen des Lebensniveaus gehen. „Mitte“ steht in dieser Weltsicht für einen sozialen Ort, der nur deshalb kein „unten“ bezeichnet, weil es in sozialer Nachbarschaft noch ein ,ganz unten“ gibt. „Mittlere Mitte“, das heißt vor allem, nach oben geht nicht mehr viel. Der Abstand zur „Oberschicht“ ist unüberwindbar. Doch nicht allzu weit entfernt von der eigenen sozialen Position lauern Ausgrenzung und Prekarität. Man mag sich ungerecht behandelt fühlen, hat aber noch immer etwas zu verlieren und kennt andere, denen es deutlich schlechter geht.

In der defensiven Grundhaltung klingt eine subjektiv erlebte Abwertung des Arbeiterstatus an. Arbeiter wird man nur, sofern sich keine andere Option bietet. Wer kann, „geht ins Büro oder studiert“ (Dörre et al. 2013b). Auch diejenigen, die sich bewusst als Arbeiter bezeichnen, ziehen daraus überwiegend kein positives Selbstbewusstsein. Sie verstehen sich als „,normal“ (Pro3), werden jedoch von der Wahrnehmung getrieben, in einer Gesellschaft mit dynamischen Arbeitsmärkten, in der scheinbar alles beständig besser wird, festzustecken. Arbeiterinnen erleben den Rückgang der Arbeitslosigkeit und glauben dennoch nicht, dass sich in ihrem eigenen Leben Grundlegendes verbessern wird. Das dichotome, aber keineswegs bipolare Bewusstsein erinnert vordergründig an jene „Schicksalsgemeinschaft“, die Popitz, Bahrdt und Forschungsgruppe für die Bundesrepublik der späten 1950er Jahre beschrieben haben. ,Schicksalsgemeinschaft“" bezeichnete damals kein stigmatisiertes „unten“, dem es zu entrinnen galt, sondern ein Kollektiv, dessen zugehörige 
Arbeiter nur gemeinsam aufsteigen konnten. Weil Aufstieg ausschließlich als einer der gesamten Arbeiterschaft vorstellbar war, konnte die dichotome Weltsicht zum universell verwendbaren Deutungsschema werden, das von der Macht des Kapitals über die Kriegsgefahr bis hin zur Ohnmacht der Arbeiter alles auf einen Nenner zu bringen in der Lage war (Popitz et al. 1957, S. 234f.). Im Kontrast dazu finden wir bei den von uns befragten Arbeiterinnen eine Dichotomie mit dem Zusatz eines abschreckenden ,tief unten“. Sich trotz niedriger Löhne der sozialen Mitte zuzurechnen, bedeutet auch, mit den besonders prekären Lagen ,ganz unten“ nichts zu tun zu haben. Der so modifizierten dichotomen Weltsicht ist die gemeinsame Aufstiegsperspektive und damit auch die Deutungshoheit für Ereignisse in der ,großen Welt" abhanden gekommen. Hierarchische (Klassen-)Beziehungen nimmt man im Mikrokosmos des Betriebs durchaus wahr. Entsprechende Erfahrungen mögen die subjektive Sicht auf die Arbeitswelt prägen; dass sie auch die Deutung politischer Ereignisse in der geschichtsmächtigen, der politischen Welt bestimmen, ist damit nicht gesagt.

\subsection{Unverschuldet anormal - Gründe für Unzufriedenheit}

Warum verbinden sich dichotomische Weltsichten von Arbeitern mit rechtspopulistischen Orientierungen? Unsere Basisstudie hatte bereits relevante Topoi einer rechtspopulistischen Alltagsphilosophie identifiziert. Dazu zählten: eine Ablehnung von Fremden, Ausländern und Migranten insbesondere mit muslimischem Hintergrund, die häufig Nützlichkeitskriterien entsprach; aggressive Attacken gegen ,Sozialschmarotzertum“; Nationalstolz gepaart mit Geschichtsrevisionismus sowie harsche Kritik an der gesamten politischen Klasse bei gleichzeitiger Relativierung der Sinnhaftigkeit demokratischer Verfahren und Institutionen (Dörre 2006, S. $157 \mathrm{ff}$.). Parteipolitisch ließen sich rechtspopulistische Weltsichten nicht eindeutig zuordnen. Äußerungen, wonach Organisationen der extremen Rechten die „richtigen Themen“ ansprachen, deuteten jedoch die Möglichkeit einer parteipolitischen Verselbständigung bereits an. Zwar sahen wir uns nicht mit geschlossen rechten Weltbildern konfrontiert, waren aber bereits auf ein verfestigtes „System expliziter und spezifisch politischer Prinzipien“, gestoßen (Bourdieu 1988, S. 655 f.). Die Herausbildung dieser Axiomatik ließ sich nicht unmittelbar auf Unsicherheitserfahrungen zurückführen. Vielmehr handelte es sich um Haltungen, Urteile und Deutungsschemata, die gegenüber situativen Einflüssen und unmittelbaren Erfahrungen eine erhebliche Persistenz aufwiesen. ${ }^{7}$

Deshalb verwundert kaum, dass sich wichtige Topoi rechtspopulistischer Orientierungen aus der Basisstudie auch in der aktuellen Befragung wiederfinden. Das gilt zunächst für die Nationalisierung der sozialen Frage und die Tendenz zu exklusiver Solidarität. Nehmen wir als Beispiel eine Arbeiterfamilie aus einer Kleinstadt im Osten. Beide Partner sind berufstätig und arbeiten $40 \mathrm{~h}$ Vollzeit für einen Brut-

\footnotetext{
7 Für einen Zuwanderungsstopp konnte man sein, obwohl oder weil man in einer Stadt mit verschwindend geringem Ausländeranteil lebte. Die Ansicht, Migranten nähmen Deutschen die Arbeitsplätze weg, formulierten Interviewpartnerinnen, die ihren eigenen Arbeitsplatz für sicher hielten. Und hartes Durchgreifen gegen „Sozialschmarotzer“ forderten ausgerechnet Personen, die selbst wirtschaftlich saturiert waren.
} 
to-Monatslohn von 1600 bzw. $1700 €$. Nach Abzug aller Fixkosten verbleiben dem Haushalt mit zwei Kindern $1000 €$ netto, von denen Kleidung, Nahrung usw. bezahlt werden müssen. Mit diesem Budget wird jede größere Anschaffung, jede Reparatur am Auto zum Problem. Alles, was das Leben subjektiv zu einem guten macht und in der Gesellschaft als normal gilt, sei es Urlaub, sei es der Restaurantbesuch am Wochenende, kann sich die Familie trotz harter Arbeit nicht leisten. Deshalb empfindet man sich als unverschuldet anormal: ,Jeder Deutsche hat ein Grundgehalt von $3300 €$ so im Durchschnitt. Dann frage ich mich jetzt, was bin ich dann? Bin ich kein Deutscher?“ (Pro3).

Arbeiter, wie der zitierte, halten sich weder für arm noch für prekär, obwohl ihr Verdienst allenfalls knapp oberhalb des Mindestlohns liegt. Immerhin unbefristet beschäftigt, möchten sie als ,ganz normal“ gelten. Trotz aller Anstrengungen gelingt der Anschluss an die gewünschte Normalität aber nur teilweise. Das macht unzufrieden. Von seinem Lohn, so der zitierte Arbeiter, könne er „nicht leben“. Nur als findiger Ostdeutscher sei er überhaupt in der Lage, mit dem niedrigen Verdienst über die Runden zu kommen. Manchmal schlage seine Unzufriedenheit ,in Wut um“. Es müsse „,einfach mal irgendwo was passieren“: „Es wird nicht mehr allzu lange dauern, dann haben wir hier vielleicht einen Bürgerkrieg“ (Pro3). Dass sich die angesammelte Wut vornehmlich gegen Fremde richtet, offenbart eine rhetorische Frage (,Bin ich etwa kein Deutscher?“). In ihr wird das Deutschsein zur Chiffre, die den Anspruch auf ein ,normales“ Leben legitimieren soll, wie es allen Deutschen zusteht. Das war schon während der Wendezeit so. Deutscher zu sein, hieß seinerzeit, einen Anspruch auf Gleichbehandlung und Gleichwertigkeit anmelden zu können, der die Angleichung der Lebensverhältnisse in Ost und West einschloss. Dieser Anspruch ist unerfüllt geblieben. Nun wird er als exklusiver eingeklagt, weil Normalität nur für Deutsche verlangt wird. Exakt dies, die Reklamation eines exklusiven „Ortsbonus“ (Milanovic 2017, S. 123) für deutsche Arbeiterinnen, bezeichnet den Umschlagpunkt, an dem sich dichotomes Bewusstsein in Richtung einer bipolaren Innen-Außen-Abgrenzung verschiebt.

In der Basisstudie hatten wir solche Weltsichten als reaktiven Nationalismus bezeichnet. Dessen Zentrum ist die Vorstellung einer Wohlstandsinsel Deutschland, die es vor illegitimen, fremden Ansprüchen zu schützen gilt. Um den Kuchen nicht mit zu vielen teilen zu müssen, sollen die Zugänge zur Insel erschwert und scharf kontrolliert werden. Bevorzugte Ausschlusskriterien sind mangelnde wirtschaftliche Nützlichkeit und Zugehörigkeit zu einer als minderwertig klassifizierten Kultur. Innerhalb dieses Deutungsmusters wird Solidarität zu einer exklusiven Ressource. Man verhält sich durchaus solidarisch, aber bevorzugt unter seinesgleichen - unter Stammbeschäftigten des eigenen Betriebs und, das ist das Einfallstor für Rechtspopulismus, den Angehörigen der eigenen Nation. Bei aktuell befragten Arbeiterinnen kommt etwas anderes hinzu: Je geringer ihre Hoffnung ist, trotz individueller Anstrengungen und gewerkschaftlicher Kämpfe Anschluss an die prosperierende Gesellschaft zu finden, desto stärker tendieren sie dazu, wahrgenommene Verteilungsungerechtigkeit als Konflikt zwischen leistungsbereiten Inländern und leistungsunwilligen, kulturell nicht integrierbaren Ausländern zu interpretieren. 
Entsprechende Weltsichten korrespondieren keineswegs ausschließlich mit niedrigem Verdienst. Sympathien für die äußerste Rechte finden sich auch bei Beschäftigten, die überdurchschnittlich verdienen. Da ist der Facharbeiter, dessen Gesundheit unter dem Schichtsystem leidet. Da ist der Ingenieur, dessen Gehalt noch immer niedriger ist als das des West-Kollegen. Da ist der Blick auf den Lebenszusammenhang mit steigenden Mieten, bröckelnder Infrastruktur und schrumpfender Bevölkerung am Wohnort. Und da ist ein Rentenbescheid, der wegen unsteter Erwerbsbiografie ein Alterseinkommen allenfalls knapp oberhalb der Grundsicherung verheißt. Kurzum, da ist der erlebte Kontrast zu einer medial inszenierten Welt, in der die eigenen Probleme gar nicht vorkommen. Persönlich kennt man „Leute, die im gleichen Beruf deutlich weniger kriegen oder vielleicht sogar noch mehr, je nachdem, wo man eben ist" (Pro2). Als Gewerkschafter und Betriebsrat weiß man zudem um die wachsende Einkommens- und Vermögensungleichheit im Land. Deshalb ist es subjektiv kein Widerspruch, mit dem eigenen „Verdienst zufrieden“ zu sein, um dennoch festzustellen: „Die, die was zu sagen haben, das sind die mit Macht oder Geld“, und die können ihre ,[Entscheidungen] anderen einfach überstülpen“ (Pro2).

Als ungerecht gelten nicht nur materielle Verteilungsverhältnisse: „Es ist [...] eine Mischung von vielen Einflüssen, die Arbeitnehmer unzufrieden macht. Im Osten leben die meisten an Orten, aus denen man kommt und nicht in Städten, in die man geht. Man kann fest angestellt sein und verdient doch nicht genug, um sich ein Leben leisten zu können, wie es die Medien als normal darstellen. Viele haben das Gefühl, in einer prosperierenden Gesellschaft nicht mithalten zu können, den Anschluss zu verlieren. Für diese Probleme gibt es aber keine gesellschaftliche Öffentlichkeit. Arbeiter kommen nirgendwo vor. Und dann kommen die Flüchtlinge und erhalten eine Aufmerksamkeit, die man selbst nicht bekommt. Es gibt Investitionen, Lehrer, Personal für Sprachkurse und berufliche Qualifizierung. Das halten viele für ungerecht. Und deshalb ist es selbst für Betriebsräte und aktive Gewerkschafter kein Widerspruch, sich aktiv an einem Arbeitskampf zu beteiligen und gleichzeitig zur Pegida-Demonstration zu gehen“, schildert ein Gewerkschaftssekretär (Sek1) eine verbreitete Problemsicht.

Arbeiterinnen gleich welcher Einkommensklasse haben sich im Osten nach eigenem Empfinden jahrelang hinten angestellt. Nun sehen sie ihre Leistung gleich doppelt entwertet. Weder sind sie bei Lohn, Arbeitszeit und Arbeitsbedingungen auf Westniveau angelangt, noch wird ihr Beitrag von denen angemessen respektiert, die Flüchtlingen plötzlich ,alles“ geben. Nachdem es jahrzehntelang hieß, dass es vor Ort nicht mehr für alles reiche, dürfen sich, so die Wahrnehmung, nun Menschen in der Reihe der Anspruchsberechtigten vordrängeln, die nichts in die Sicherungssysteme eingezahlt haben. Nicht nur rechtsorientierte Befragte empfinden das als zusätzliche Abwertung. Wähnt man sich schon als Arbeiter und „Ossi“ am unteren Ende der Anerkennungspyramide, werden nun auch noch die Fremden bevorzugt. Selbstaufwertung mittels Abwertung anderer ist eine mögliche, für manche eine subjektiv naheliegende Reaktion. 


\subsection{Gefährliche Klassen, Ausschluss, Gewalt}

Objekt einer kollektiven Abwertung durch Arbeiterinnen, die sich selbst als abgewertet empfinden, sind bevorzugt diejenigen ,tief unten“, die sich des Verdachts ausgesetzt sehen, auf Kosten der „Normalbürger“ leben zu wollen. Nicht nur Fluchtmigranten und Langzeiterwerbslose, auch „faule Griechen“ oder andere vermeintlich Leistungsunwillige können unter Generalverdacht geraten. Verdachtsmomente sind in den gesellschaftlichen Öffentlichkeiten jederzeit präsent. Im Beobachtungszeitraum wurden sie vom ehemaligen sozialdemokratischen Politiker Thilo Sarrazin besonders wirkungsvoll in Szene gesetzt. Eine moderne Industriegesellschaft, die Klassenprivilegien abgeschafft habe, bringe, so Sarrazin, generell eine „Durchmischung ehemals stabiler Schicht- und Klassenzugehörigkeiten mit sich“: ,Während die Tüchtigen aufsteigen und die Unterschicht oder untere Mittelschicht verlassen, wurden und werden in einer arbeitsorientierten Leistungsgesellschaft nach ,unten * vor allem jene abgegeben, die weniger tüchtig, weniger robust oder ganz schlicht ein bisschen dümmer und fauler sind“" (Sarrazin 2015, S. 79f.). Das Leistungsprinzip, im Arbeiterbewusstsein seit jeher ein wichtiger Gerechtigkeitsmaßstab, wird so zu einem Selektionsmechanismus umdefiniert, der eine natürliche Bestenauslese mit klassenkonstitutiver Wirkung begünstigt. Wer unten ist, der ist es nach dieser Logik zu Recht. Wenn dann jedoch die genetisch wie kulturell bedingt weniger Intelligenten - unter ihnen vornehmlich integrationsunwillige Einwanderer aus Afrika, dem Balkan und der Türkei - mehr Kinder bekommen als die Intelligenten, muss das nach Sarrazin unweigerlich zu einem Verlust an wirtschaftlicher Leistungsfähigkeit führen.

Von uns befragte Pegida/AfD-Anhängerinnen fügen dem ein weiteres Problem hinzu. Sie betrachten die ethnisch heterogene Unterklasse als Sicherheitsrisiko. Zwar räumen die Befragten ein, dass der Ausländeranteil in der eigenen Region nicht sonderlich hoch sei, fügen jedoch hinzu: „Ich sage mal, es ist ja auch gut, dass es so ist. Weil wenn diese Bewegung [Pegida] hier nicht wäre, würde es vermutlich auch anders aussehen. Dieser Kulturkreis [...], die wollen ja unter sich sein, unter ihresgleichen und das haben sie dann eben in Großstädten wie Stuttgart, München, Berlin schon eher als in Elbestadt. Deswegen, denke ich, hat die Bewegung schon dazu geführt, dass viele den Weg hierher meiden [...]. Es gab auch eine Zeit, da ist es mehr aufgefallen, speziell auch meiner Freundin, weil die gerne mal [...] unterwegs ist zum Shoppen und da dann auch vermehrt solche Truppen Jugendlicher aufgetreten sind, die offenbar anderer Herkunft waren und dann eben meine Freundin, ja, schon belästigt haben und angesprochen haben wie von wegen: Heirate mich und hübsche Frau und sowas, was schon sehr nervig und penetrant ist. Das gehört sich einfach nicht“" (Pro 2).

Im Statement wird Unterklasse als „Kulturkreis“ identifiziert, für den Regellosigkeit und Anmache charakteristisch sind. Selbstverständlich wollen die vor Ort kaum präsenten Flüchtlinge unter sich sein, um ihre Kultur zu leben. Deshalb zieht es sie in die Metropolen. Doch das - gegenwärtig nur fiktive - Sicherheitsrisiko für Einheimische und besonders für ,unsere Frauen“ kann jederzeit neu entstehen. In der Beschwörung einer Gefahr durch kulturfremde Männer, die ihre Triebe angeblich nicht im Griff haben, legitimiert sich ein tradiertes Männerbild neu. Die 
Gefahr unzivilisierter Unholde vor Augen, präsentieren sich deutsche Männer als Beschützer ,,ihrer“ Frauen und legitimieren so eigene patriarchale Besitzansprüche. Die Beschwörung von Gefahren durch die neuen gefährlichen Klassen leistet zugleich, was Robert Castel mit seinem Hinweis auf zwei grundlegende Sicherungssysteme, den Wohlfahrts- und den Rechtsstaat (Castel 2005, S. 7), als Möglichkeit bereits angedeutet hatte. Über die Diabolisierung der Unterklassen verschiebt sich der gesellschaftliche Sicherheitsdiskurs. Verbreitete Sorgen um soziale Sicherheit, die auch auf real Erlebtem beruhen können, schlagen in ein Bedürfnis nach Schutz vor der Bedrohung durch unzivilisierte Eindringlinge aus den gefährlichen Klassen um.

Gegen das diabolisierte Andere hilft nach Auffassung rechter Arbeiter nur hartes Ab- und Ausgrenzen. Dem Ruf nach öffentlicher Sicherheit entspricht der Wunsch nach Bewahrung des eigenen Lebensstils. Um letzteren zu erhalten, ist den Befragten mit AfD/Pegida-Sympathien nahezu jedes Mittel recht. Rechte Arbeiter neigen diesbezüglich zu einer Haltung, die in ihrer Radikalität und Eindeutigkeit überrascht: „Flüchtlinge müssten also meiner Meinung nach [...] raus. Wer hier jetzt herkommt, arbeitet, sich integriert, wer sich einordnet, unterordnet, kein Thema. Da habe ich ja nichts dagegen. Aber die, die nur hierher kommen und die Hand aufhalten und sich benehmen wie das Letzte und denken, die können sich alles erlauben, raus! Ich meine, das klingt zwar vielleicht blöd oder hart. Ich hätte kein Problem damit, jetzt mal Buchenwald wieder aufzumachen, einen Stacheldraht ringsrum, die dort rein, wir dort draußen. Dann kommt sich keiner in die Quere. Und die dort so lange drinnen lassen, alles natürlich normal, human [...] und werden dann abgeschoben, fort. Gar nicht irgendwie, dass irgendwas passieren kann“ (Pro3).

Sicher ist dieses Plädoyer für ,normale“, „humane“ Konzentrationslager, in welchen Flüchtlinge ,nur“ interniert, aber nicht verbrannt werden sollen, eine extreme Ausnahme. Doch bei genauerer Prüfung sagt der zitierte Betriebsrat lediglich, was andere eleganter ausdrücken. Für die Unterbringung in Sammelunterkünften oder Asylzentren auch außerhalb Deutschlands seien lange nach der AfD nun auch die etablierten Parteien, stellen andere Befragte süffisant fest (Pro2; Pro4). Sie lassen keinen Zweifel daran, dass der Aufenthalt für Ankömmlinge, die sich in ihrer Mehrheit nur ,die sozialen Vorteile“ zu eigen machten, möglichst unkomfortabel zu gestalten sei. Es genüge völlig, im Auffanglager Essen, Trinken und Schlafgelegenheiten zu bieten: „Wofür brauchen die [...] ein Taschengeld? Wer sich etwas verdienen will oberhalb dem, was es gibt, der muss dafür auch etwas tun. Weil niemandem hier fällt irgendwas zu“"(Pro2).

Wird das Leistungsprinzip durch eine angebliche Bevorzugung Geflüchteter außer Kraft gesetzt, rechtfertigt das aus Sicht radikal rechter Arbeiterinnen Gegenmaßnahmen. Bezeichnend ist, dass alle Befragten, die mit Pegida, der AfD oder extremen Rechtsparteien sympathisieren, eine erstaunliche Gewaltaffinität aufweisen. Keiner dieser Arbeiter will sich eindeutig und ohne jede Relativierung von Gewalttaten gegen Flüchtlinge oder deren Unterkünfte distanzieren. Standardargument ist der Hinweis, es gebe Verrückte und Gewalttäter auch auf der Linken (Pro1). Ebenfalls beliebt sind Ausweichargumente. So sei die Holocaust-Leugnung seitens des Pegida-Redners Akif Pirinçci ,schon ein sehr grenzwertiger Auftritt“ gewesen, heißt es. Doch wenn jemand, der aus dem muslimischen Kulturkreis komme, ,über seine ei- 
genen Leute kritisch redet, kann es nicht ganz verkehrt sein“ (Pro2). Als wichtigste Legitimation dient ein Notwehrargument: „Weil Gewalt erzeugt auch Gegengewalt, also es passieren sehr viele Zwischenfälle mit Ausländern. Das wird kleingeredet. Das interessiert keinen. Und ich meine, wenn meiner Familie jetzt was passieren würde mit so einem Ausländer, ich würde dann auch im Prinzip Gewalt anwenden. Man muss sich doch wehren. Man kann sich doch nicht nur ducken und die Backen hinhalten“ (Pro 3).

\section{Ansichten zu Demokratie, Volk und System}

Das Notwehrargument soll relativieren, was bei Anschlägen auf Geflüchtete geschehen ist. Vergleichbare Aussagen hatte es im Rahmen unserer Basiserhebung nicht gegeben. Wir konnten davon ausgehen, dass sich Rechtspopulismus durch eine Ablehnung gewaltsamer Systemveränderung vom Rechtsextremismus abhob. Diese Unterscheidung ist nun brüchig geworden.

\subsection{Mehr Demokratie wagen}

Es gibt einen weiteren Topos, an dem sich die Befunde aus Basis- und Primärerhebung/Tiefenbohrung erheblich unterscheiden. Das betrifft die Haltung befragter Pegida/AfD-Sympathisanten zur Demokratie. Charakteristisch ist nun nicht mehr die Abwertung, sondern die offensive Vereinnahmung von Demokratie. Viele Befragte mit Affinität zum Rechtspopulismus plädieren für mehr direkte Demokratie: „Na, für mich wäre erstmal eine gute Demokratie, wenn wir eine Volksabstimmung hätten, dort müsste man anfangen. Und das Zweite wäre für mich ein ordentliches Strafgesetz. Volksabstimmungen, wo man sieht, wo die Stimmung hingeht von den Menschen im Land, dass nicht irgendein Politiker sich anmaßt: Ich entscheide das jetzt mal für alle, oder so" (Pro1). Befragte rechte Arbeiter könnten sich eine Demokratie nach Schweizer Vorbild gut vorstellen. Allerdings reduzieren sie Demokratie auf ein Mehrheitsprinzip. Das Volk soll unmittelbar herrschen und dem Volkswillen auf direktem Weg zum Durchbruch verhelfen. Volk ist in diesem Denken identisch mit gesundem Menschenverstand. Kann sich das populare Vernunftprinzip unverfälscht äußern, steht fest, dass sich die ,richtigen“ Auffassungen durchsetzen werden. Härtere Strafen für Mörder und Vergewaltiger wären dann eine Selbstverständlichkeit. So jedenfalls das Kalkül, welches einem identitären Demokratieverständnis nahezu passgenau entspricht. Demokratisches Subjekt ist éthnos, ein homogenes Volk von Biodeutschen, das autonom über seine Geschicke entscheidet und abgehobene Eliten samt „Systemparteien“ zur Ordnung ruft. Diesem Demokratieverständnis sind Völker- und Menschenrechte im Zweifelsfall verzichtbar, es zählt das Prinzip ,Deutsche zuerst".

Dennoch wäre es zu einfach, hinter den Demokratisierungswünschen der Befragten lediglich Ressentiments und Rassismus zu vermuten. Rechte wie linke Arbeiterinnen schildern eine Vielzahl von Entscheidungen, die, angefangen bei der Bankenrettung während der Finanzkrise, über die Köpfe der Befragten hinweg getroffen wurden. Auch die humanitäre Entscheidung der Regierung Merkel, eine große Zahl 
Geflüchteter unter Aussetzung gültiger Einreisebestimmungen aufzunehmen, fällt subjektiv unter diese Kategorie. Im Kontrast dazu werden Bewegungen wie Pegida als Demokratisierungsimpuls erlebt. Selbst linke Gewerkschaftssekretärinnen hegen keine Zweifel, dass es dem Gros der Pegida-Demonstranten primär darum geht, zum Subjekt politischer Entscheidungen zu werden: „Da artikuliert sich so ein: Ich will jetzt auch mal ein Stück vom Kuchen. Habe ich nie gehabt, haben meine Eltern nicht gehabt, haben meine Großeltern nicht gehabt, die Region, in der ich hier rumhänge, ist deindustrialisiert worden, die Gewerkschaften sind mäßig durchsetzungsfähig [...]. Und ich will jetzt auch mal. Und dann ist eine Bewegung, die Woche für Woche größer wird, natürlich etwas, woran man sich orientiert und sagt, Mensch, da geht was. Egal was erstmal, aber dieses Gefühl, da ist endlich mal was, da werden wir es jetzt den Herrschenden mal zeigen. Da geht es ganz wenig um Inhalte, sondern ganz viel um Emotionen. Da geht es darum, wahrgenommen werden zu wollen“ (Sek2). Unzufriedene stören sich allerdings wenig daran, dass sie bei den PegidaDemonstrationen Repräsentanten der äußersten Rechten an ihrer Seite finden.

\subsection{Der Betrieb als demokratiefreie Zone und Ort widersprüchlicher Erfahrung}

Für das Demokratieverständnis rechtspopulistischer Arbeiter ist bedeutsam, dass die Betriebe - nach Einschätzung der Gewerkschaftssekretärinnen in Sachsen und im Osten besonders ausgeprägt - mehrheitlich mitbestimmungsfreien Zonen ähneln. Partizipative Mitbestimmungskulturen sind noch immer Ausnahmeerscheinungen. Wo Betriebsräte neu gegründet oder tarifliche Normen durchgesetzt werden sollen, geschieht das teilweise in hartnäckigen Häuserkämpfen, von Betrieb zu Betrieb und von Unternehmen zu Unternehmen. Handlungsfähig sind Gewerkschaften nur, wo sie im Unternehmen über Mitglieder verfügen. Betriebliche Basisinitiativen sehen sich jedoch mit hartem Arbeitergeberdruck bis hin zu professionellem union busting ${ }^{8}$ konfrontiert. Konflikte um basale Partizipationsrechte bis hin zur „Hexenjagd gegen den Betriebsratsvorsitzenden“ (Sek8) sind an der Tagesordnung. Demokratie ist deshalb ,nichts Einfaches“. Im Kontext betrieblicher Herrschaft ist direkt erfahrbar, dass sie ,wirklich weh tut, wenn man es ernst meint“ (Sek8). Ohne selbsttätiges Engagement von Beschäftigten und Gewerkschaftsmitgliedern sind in der fragmentierten Arbeitswelt weder Mitbestimmungsrechte noch tarifliche Normen durchsetzbar. Das bleibt auch rechtsorientierten Arbeiterinnen nicht verborgen, sobald sie sich in der betrieblichen Arena betätigen.

Ein gutes Beispiel liefert die Auseinandersetzung um einen Logistikbetrieb, dessen Belegschaft zu 50\% aus polnischen und tschechischen Leiharbeitern besteht. Leiharbeitskräfte wurden eingestellt, weil sie als „Sachkosten“ zählen und deshalb das Personalbudget und die Gewinnmarge des Unternehmens nicht belasten. Die Erfahrung der Festangestellten mit den Leiharbeitern ist widersprüchlich. Einerseits gibt es „wirklich gute Kollegen“, die „Deutsch sprechen, die arbeiten können und dann hast du eben auch welche, wo du sagst, die kann ich als Chef jetzt mal

\footnotetext{
${ }^{8}$ Gemeint ist der Einsatz professionell arbeitender Rechtsanwälte, die sich auf die Bekämpfung von Betriebsräten und Gewerkschaften spezialisiert haben (Dörre et al. 2017, S. 123-142).
} 
in eine Firma nicht reinholen“ (Pro3). Eigentliches Problem sind weniger fehlende Sprachkenntnisse als die Tatsache, dass Festangestellte ,mit den Leuten alleine gelassen [werden]: ,Sieh zu, wie Du den anlernst”, mehr nicht!“ (Pro3). Im Kampf um einen Anerkennungstarifvertrag galten die Leiharbeiter anfänglich als potenzielle Streikbrecher. Gespräche mit dem rechtsorientierten Betriebsrat führten jedoch zu einer solidarischen Haltung der prekär Beschäftigten. Einen drohenden Arbeitskampf vor Augen, lenkte die Geschäftsführung des Mutterunternehmens überraschend ein und reintegrierte den zuvor outgesourcten Betrieb. Im Ergebnis erhielt jedes Belegschaftsmitglied einen Tariflohn und damit ein monatliches Plus von einigen hundert Euro. Den Erfolg verdankt die lokale IG Metall auch jenem Betriebsrat, der Flüchtlinge in Lagern sehen will. Der Befragte ist noch immer radikal rechts. Doch er hat gelernt, dass es Solidarität unter Arbeiterinnen verschiedener Nationen geben kann und setzt sich für die Übernahmen der Leiharbeitskräfte ein: „,Das hätte der Typ sich vor einem Jahr nicht denken lassen, dass der sich da mal Gedanken darüber macht, dass er sich um den Arbeitsplatz von Ausländern kümmert. Aber irgendwas ist da passiert in seinem Kopf“"(Sek2).

Das Beispiel veranschaulicht den Stellenwert, den Arbeitserfahrungen innerhalb der rechtspopulistischen Weltsicht besitzen. Es handelt sich um Erfahrungen in der „kleinen Welt“ des Betriebs, die von der strukturellen Widersprüchlichkeit marktwirtschaftlich-kapitalistischer Produktion geprägt werden. Einerseits sollen die Arbeits- und Lebensbedingungen möglichst flexibel und die Arbeitskräfte möglichst mobil gehalten werden, um sie den Produktionserfordernissen optimal anzupassen. Andererseits erweisen sich Festangestellte mit Bindung an das Unternehmen häufig als besonders leistungsfähig (Balibar 1990, S. 256). Diese strukturelle Widersprüchlichkeit reproduziert immer wieder rassistisch-nationalistische und sexistische Diskriminierungen. Wie am Beispiel des Logistikbetriebs gezeigt, kann sie aber auch zur Quelle gegenläufiger Erfahrungen werden und zur Überwindung ethnisch-nationaler Spaltungen motivieren.

\subsection{Gesellschaftsbild ohne Gesellschaft}

Solidaritätserfahrungen in der ,kleinen Welt“ des Betriebs reiben sich an verfestigten Deutungen der ,großen Welt“. Geht es um politische Konflikte und Großereignisse, operieren rechte Arbeiterinnen mit einer schlichten Bipolarität. Dem System, einer abstrakten Ansammlung dunkler Mächte, wird ein Volk gegenübergestellt, das idealerweise nicht von Interessengegensätzen und Egoismen zerrissen wird. Auch die Sicht rechter Lohnabhängiger auf die ,große Welt" hat sich im Vergleich zur Basisstudie erheblich verändert. Rechtspopulistische Orientierungen fächerten sich seinerzeit in konformistische, konservierende und rebellische Varianten auf. Rebellische Orientierungen fanden sich vor allem bei Erwerbslosen und prekär Beschäftigten. Ihnen diente das negativ besetzte Bild des Anderen dazu, positiv besetzte Zugehörigkeiten über eine Abwertung anderer, noch schwächerer Gruppen oder Individuen zu konstruieren. Auf die konservierende Variante stießen wir bei formal gut integrierten Beschäftigten, die versuchten, ihre eigene soziale Position zu verteidigen, indem sie Ressentiments zur Triebfeder, gesellschaftlicher und politischer Aktion“ (Castel 2005, S. 67 f.) machten und ,die Verantwortung für das eigene Un- 
glück bei jenen Gruppen“ suchten, ,die sich auf der sozialen Leiter knapp oberhalb oder knapp unterhalb der eigenen Position“ befanden (ebd., S. 68). Ein konformistischer Rechtspopulismus, der wesentlich auf Überanpassung an hegemoniale Normen beruhte, machte sich bevorzugt in höheren, abgesicherten beruflichen Positionen bemerkbar. Charakteristisch für diese Variante war, dass sie Team, Belegschaft und Nation als Gemeinschaften hart arbeitender Menschen konstruierte. Was sie von sich selbst erwarteten, die vorbehaltlose Erfüllung vorgegebener Leistungsnormen, verlangten konformistische Angestellte auch von anderen. Integration von Migranten war für sie daher nur als einseitige Anpassung an die „deutsche Kultur“ der Leistungsbereiten vorstellbar.

In der aktuellen Erhebung hat sich das rebellische Moment verallgemeinert. Es findet sich auch bei überdurchschnittlich gut verdienenden Beschäftigten in attraktiven beruflichen Positionen. Alle Befragten, die mit der populistischen Rechten sympathisieren, stellen die Systemfrage: „Ist ja nicht so, dass die, die auf die Straße gehen [...] alles Nazis sind. Die gehen halt auf die Straße, weil irgendwas mit dem System nicht stimmt“ (Pro1). System fungiert subjektiv als Sammelbezeichnung für vieles - für die Finanzwirtschaft, die Europäische Union, das transnationale Euro-Geld, die politische Klasse, die Eliten, das Kapital, die Manager, aber auch für ,,abgehobene Gewerkschaftsbonzen“ (Sek1). Beim Kapital ist der Unterschied zwischen national und international überraschenderweise von untergeordneter Bedeutung. Den Beschäftigten sei ,egal, wo die Kohle“ herkomme: „Also da ist [...] dann das Kapital aber auch national ganz schnell wieder ein Schwein, wenn hier zu wenig Kohle ankommt. Aber wenn ich hier einen Job mit relativ guten Verhältnissen habe, ist alles andere völlig egal [...]. Ob das ein Chinese ist, der bezahlt, ob das eine staatliche Firma ist, die da Anteile drin hat, ob das ein Privater ist, das ist denen [den Arbeiterinnen, d. A.] dann völlig egal“" (Sek1).

Auf seltsame Weise entspricht das System der menschlichen Neigung, egoistisch nach Geld und Macht zu streben. Gut wäre aus Sicht der Befragten, wenn den Egoismen Grenzen gesetzt würden. Gelänge dies, könnte sich der Volkswille optimal entfalten. Für radikal rechte Arbeiter ist das Volk eine kulturell begründete Gemeinschaft. Eine solche erwünschte „Volksgemeinschaft“" habe - ein Teil der Befragten weiß es nur von den Eltern - ansatzweise in der alten DDR existiert. Selbstverständlich möchte niemand diese DDR zurück. Aber eine Gemeinschaft, die noch nicht von Egoismen, Vorteilsstreben und Ellenbogenmentalität zerstört ist, steht an oberster Stelle individueller Wunschlisten: „Ich kenne ja die DDR selber nicht mehr persönlich. Aber wenn man so die Eltern reden hört, [...] der Zusammenhalt war mehr da. Es ging mehr ums Persönliche, ums Menschliche, und nicht um: wie kann ich am besten noch mehr Geld oder irgendwas bekommen, damit [...] ich dies und das mir leisten kann“ (Pro2).

So wie sich Arbeiterinnen im Westen den idealisierten Sozialkapitalismus der alten Bundesrepublik zurückwünschen, sehnen sich rechte Gewerkschafterinnen im Osten nach der ebenfalls mystifizierten Volksgemeinschaft des „,arbeiterlichen“ DDR-Paternalismus (Engler 2004). Allerdings handelt es bei den rechtspopulistischen Weltsichten im Grunde um Gesellschaftsbilder ohne Gesellschaft. Gesellschaft ist als Kategorie subjektiv unbedeutend. Relevant ist allein die Bipolarität von Volk und System. Auch dieser Dualismus markiert einen wichtigen Unterschied zum di- 
chotomischen Arbeiterbewusstsein frühere Epochen. Auf den ersten Blick scheinen die Unterschiede nicht sehr groß. Für radikal rechte Arbeiter gibt es durchaus ,eine kleine Oberschicht, dann kommt ganz, ganz lange nichts und dann kommt unten das Fußvolk" (Pro3). Während dichotomes Klassenbewusstsein jedoch das Wissen um soziale Mechanismen adaptieren kann, welche die Armut der Armen kausal zum Reichtum der Reichen in Beziehung setzen (Wright 1985, S. 38), präferieren rechte Arbeiterinnen ein anderes Verbindungsprinzip. Für sie liefert den Kausalmechanismus, der zwischen zerstörerischem System und gebeuteltem Volk vermittelt, die Verschwörungstheorie. Gleich, um welche Problematik es sich handelt, stets sind dunkle Mächte am Werk, die dem deutschen Volk Böses zufügen wollen.

Die subjektive Relevanz von Verschwörungstheorien offenbart ein doppeltes Dilemma. Offenkundig sind die Ursachen wachsender Vermögens- und Einkommensungleichheiten derart komplex und die Entscheidungszentren, die Unsicherheit und Ungleichheit steigern, insbesondere von betrieblichen Erfahrungsräumen so weit entfernt, dass sich verschwörungstheoretische Konstruktionen als Deutungssicherheit stiftende Komplexitätsreduktionen geradezu aufdrängen. Das kann geschehen, weil Deutungsmuster, die Kausalitäten über Ausbeutung, Entfremdung und Klasse herstellen und so moralische Bindekraft entfalten, im Alltagsbewusstsein radikal rechter Arbeiter nicht mehr vorkommen.

\section{Die national-soziale Gefahr und wie ihr (nicht) zu begegnen ist}

Was bedeuten die empirischen Befunde für die eingangs aufgeworfene Kontroverse? Nachfolgend beschränken wir uns auf fünf Überlegungen.

Erstens zeugt unser Material von einer ernst zu nehmenden national-sozialen Gefahr. Der „Saatboden für einen neuen Faschismus“ (J. Habermas) existiert tatsächlich. Er entsteht, weil die völkische Rechte soziale Verwerfungen erfolgreich als Mobilisierungsressource zu nutzen vermag. In unterschiedlichen Ausprägungen findet die von ihr betriebene Ethnisierung des Sozialen Anhängerinnen auch unter gewerkschaftlich Aktiven und Betriebsräten, wenngleich große Datensätze diese Tendenz noch nicht abbilden (Hilmer et al. 2017). Sprachduktus und Begriffswahl der Befragten bewegen sich auf dem Niveau von „Urteilen zweiten Grades“, d.h. sie folgen bereits der Linie einer Partei (Bourdieu 1988, S. 656). Wer derart hermetisch argumentiert, lässt sich kaum von seinen Überzeugungen abbringen. Für jede kritische Nachfrage haben rechtspopulistische Gewerkschafter passende Antworten parat. ${ }^{9}$ Eigene Überzeugungen werden proaktiv gegen jedwede Kritik immunisiert. Gemeinsam mit der Gewaltaffinität deutet das auf eine Verfestigung und Radikalisierung rechter Orientierungen hin.

\footnotetext{
9 Das trifft nicht auf alle Befragten zu, die mit Formationen der neuen Rechten sympathisieren. Im Sample findet sich auch der qualifizierte Angestellte mit Greenpeace-Mitgliedschaft, der sich wegen der Migrationsströme um den gesellschaftlichen Zusammenhalt sorgt und deshalb AfD wählt. Und es gibt den Betriebsrat, der es lange mit der Linkspartei hielt, jedoch deren Migrationspolitik kritisiert und deshalb nun die rechtspopulistische Formation favorisiert. Bei diesen Befragten, die überwiegend linke Positionen formulieren, kann von einer rechtspopulistischen Axiomatik nicht oder noch nicht gesprochen werden.
} 
Wir sprechen von einer national-sozialen Gefahr, weil das zusätzlich zur Betonung der sozialpopulistischen Dimension die Offenheit für NS-Ideologeme kenntlich machen soll. Mit der Bezeichnung völkisch-populistisch verbinden wir eine ähnliche Intention. Vorstellungen vom Volk als Gemeinschaft sind der ideologische Kitt, der radikal rechte Weltbilder zusammenhält. Beide Zuschreibungen signalisieren, dass Arbeit am Begriff notwendig ist. Wenn die Befürwortung von Gewalt als Kriterium wegfällt, um Rechtspopulismus und -extremismus voneinander zu unterscheiden und die Abgrenzung von traditionsfaschistischen Positionen für die neue Rechte bis $\mathrm{zu}$ einem gewisse Grade obsolet wird, muss das zu bezeichnende Phänomen neu benannt werden. Um das zu betonen, spricht Wilhelm Heitmeyer von einem ,,autoritären Nationalradikalismus“ (Heitmeyer 2018). Man mag darüber streiten, ob das eine angemessene Begriffswahl ist. In jedem Fall trifft zu, dass die Radikalisierung rechter Orientierungen demokratietheoretischen Auffassungen widerspricht (Mény und Sure 2002; Patzelt und Klose 2016), denen zufolge eine Parlamentarisierung des Rechtspopulismus zu dessen Einhegung und Befriedung beiträgt. In Sachsen hat die NPD über zwei Legislaturperioden hinweg im Landtag gesessen. Wo sie, wie in unseren Untersuchungsregionen, ihre größten Wahlerfolge erzielte, dominiert nun die AfD. Parlamentarische Präsenz der äußersten Rechten trägt offenbar dazu bei, dass sich die Grenzen des Sagbaren in der Zivilgesellschaft immer weiter nach rechts verschieben (Göttinger Institut für Demokratieforschung 2017; Zick und Küpper 2015). Selbst Tabubrüche wie die Holocaustleugnung schrecken AfD/ Pegida-Sympathisanten nicht mehr ab. Gewalt gegen Geflüchtete wird als Ausdruck berechtigten Volkszorns relativiert. Was anderswo nur stillschweigend gedacht und geduldet wird, äußert sich in unserer Schwerpunktregion unverblümt - und dies selbst im Kreis gewerkschaftlich Aktiver.

Zweitens besteht kein Zweifel, dass das betriebliche und gewerkschaftliche Engagement radikal rechter Arbeiterinnen von legitimen sozialen Protestmotiven getrieben wird. Dennoch handelt es sich bei den Formationen, mit denen diese Arbeiterinnen sympathisieren, nicht um Repräsentationen einer neuen Arbeiterbewegung. Arbeiterbewegungen des Marx'schen Typus sind Ausdruck eines Klassenhandelns, das auf eine Verbesserung kollektiver Positionen im sozialen Raum zielt. Solche Klassenbewegungen brechen am Kausalmechanismus Ausbeutung oder schwächer: an ungerechten Verteilungsverhältnissen auf und richten sich gegen die aneignenden Klassen. Bewegungen Polanyi'schen Typs klagen hingegen primär Schutz vor marktgetriebener Konkurrenz ein (Polanyi 1995). Sie richten sich gegen eine diffuse Marktmacht, die unter Lohnabhängigen eine Tendenz bestärken kann, ,klassenunspezifische Grenzen abzustecken, um vor dem Mahlstrom des Marktes geschützt zu werden“" (Silver 2005, S. 41). Pegida und die AfD stehen für solche Bewegungen Polanyi'schen Typs. Anstelle von Ausbeutung agiert diese Bewegung mit Kausalmechanismen wie „Umvolkung“ oder ,Einwanderung in die Sozialsysteme“. Die Motive, die zur populistischen Revolte führen, lassen sich indes nicht säuberlich in sozioökonomische und kulturelle aufspalten. Radikal rechte Arbeiter verteidigen ihren Lebensstil. Doch die verinnerlichten Dispositionen und Geschmacksurteile, die diesen Lebensstil hervorbringen, wurzeln in sozioökonomischen (Klassen-)Verhältnissen. Grundlegend für die Weltsicht der Befragten ist das Empfinden, am gesellschaftlichen Wohlstand nicht angemessen beteiligt zu sein - materiell wie kul- 
turell. Deshalb haben sich Befragte gewerkschaftlich organisiert und in Betriebsräte wählen lassen. Ihren Sozialprotest stark zu relativieren oder gar infrage zu stellen (van Dyk et al. 2017), liefe deshalb darauf hinaus, empirische Fakten zu ignorieren.

Der oft gehörte Einwand, beim Gros der AfD-Wählerschaft handele es sich nicht um Arbeiter, weshalb Gerechtigkeitsmotive nachgeordnet zu behandeln seien, führt ebenfalls nicht weiter. ,Arbeiter' ist eine analytisch höchst unscharfe Kategorie, die in unserem Sample heterogene Lagen abdeckt. Vom Leiharbeiter in der verarbeitenden Industrie über die Niedriglohnbezieherin im Online-Handel und die Erzieherin in einer Kindertagesstätte bis hin zur festangestellten Fachkraft in der Exportwirtschaft können sich Angehörige höchst unterschiedlicher Lohnabhängigengruppen subjektiv dem Arbeiterstatus zuordnen. Daran zeigt sich, dass klassische ArbeiterDefinitionen, die auf abhängige, manuell verrichtete Arbeit, institutionelle Regulationen und soziokulturelle Identitäten abheben (Schmidt 2015, S. 13), heute wenig aussagekräftig sind. Die Arbeiter-Kategorie kann so ausgedehnt werden, dass sie mit dem arbeitenden Volk nahezu identisch ist. Deutlich enger gefasst, bezeichnet sie jenes Drittel der Bevölkerung, das in der Bundesrepublik statistisch noch immer Arbeiterklassenlagen zuzurechnen ist. ${ }^{10}$ Doch ungeachtet solcher Unschärfen gehört Lohn- und Einkommensungleichheit selbst für gut verdienende Lohnabhängige zu den wichtigsten Einschränkungen selbstbestimmter Lebensführung (Wilkinson und Pickett 2009, S. 301). Man opfert „Basisgüter wie Muße“, um diese Einschränkung individuell zu überwinden (Skidelsky und Skidelsky 2014, S. 260). Da die Gewerkschaften nur begrenzt durchsetzungsfähig sind und die politische Linke mit ihren Deutungsmustern bei vielen unserer Befragten als ernst zu nehmende Alternative gar nicht erst in Betracht kommt, kann die völkische Rechte sich als Adressatin verletzten Gerechtigkeitsempfindens empfehlen. Eine Stimme für die AfD gilt subjektiv als härtest möglicher Schlag gegen ein selbstzufriedenes Establishment, zu dem man teilweise auch die Gewerkschaftsspitzen rechnet. Die Attraktivität des völkischen Populismus beruht vor allem auf dessen symbolischer Kraft. Alltagsweltlich etabliert er Kausalmechanismen, die das eigene Wohlergehen mit der Abwertung anderer verknüpfen. Solche Mechanismen lassen sich individuell leicht handhaben. Anders als solidarisches Klassenhandeln benötigen sie keine gemeinsame Aktion der Subalternen. In Akten der Selbstüberhöhung können sie subjektiv entlasten, und ihre Betätigung erscheint, sofern damit erfolgreich ins politische System hineingewirkt wird, durchaus als Akt rationaler Interessendurchsetzung (Jörke und Nachtwey 2017).

Dennoch ist der Aufstieg der völkischen Rechten drittens kein unaufhaltsamer. Ihm kann Einhalt geboten werden, sofern es gelingt, die alltagsweltliche Attraktivität des völkischen Abwertungsmechanismus zu erschüttern. Manch kritisch gemeinter Deutungsversuch läuft hingegen unbeabsichtigt auf das Gegenteil hinaus. Warum das so ist, kann das Bild von der Kristallglocke veranschaulichen, die in den Worten

\footnotetext{
$1033 \%$ im Westen, 35\% im Osten, die „Arbeiterelite“ aus Vorarbeitern, Meistern und Technikern eingeschlossen (Destatis 2016; Daten für 2014). Bezieht man die subjektiven Zugehörigkeiten ein, ist die Arbeiterschicht im Westen deutlich kleiner als sie es nach struktureller Klassenzugehörigkeit wäre (2014: $23 \% ; 1990: 27 \%)$, im Osten wäre sie nur unwesentlich größer (2014: 36\%). Vor allem für den Osten der Republik ist dieser Wandel dramatisch, denn 1990 hatten sich noch $57 \%$ der Arbeiterschicht zugerechnet (Destatis 2016, S. 206).
} 
Peter Sloterdijks die „Komfortzone“ des reichen Nordens von den Ausgeschlossenen des darbenden Südens trennt (Sloterdijk 2006). Der völkische Populismus übernimmt dieses Bild und spricht der fiktiv höherwertigen Kultur das Recht zu, die „Komfortzone“ gegen fremde Eindringlinge niederer Zivilisationsstufen zu verteidigen. Zu Recht plädieren Kritikerinnen dieser Auffassung dafür, die Protestmotive im Inneren der Kristallglocke ernst zu nehmen. Für Wolfgang Streeck verhalten sich die „kleinen Leute“ so wie die französischen Parzellbauern zu Zeiten Louis Bonapartes des III. Unfähig sich als selbstbewusste Klassen zu konstituieren, delegieren sie ihre Interessen an autoritäre Führer, die nur führen können, weil sie die Interessen der Subalternen zumindest partiell respektieren (Marx 1960, S. 111-207). Dieser von zahlreichen Marxisten immer wieder bemühten, aber nur in Teilen plausiblen Analogie (Deppe 2018) verleiht Streeck jedoch eine fatale Wendung, wenn er „Ethnonationalismus“ als Kampfbegriff enttarnen will, der eine „moralische Denunziation von Forderungen nach nationaler Politik zum Schutz gegen Risiken und Nebenwirkungen der Internationalisierung" leisten soll (Streeck 2017, S. 262f.). Facharbeiterinnen sind keine Parzellbauern. Wenn sie, obwohl gewerkschaftlich organisiert und als Betriebsräte aktiv, ihre Interessen an die äußerste Rechte delegieren, so ist das auch, aber eben nicht ausschließlich Sozialprotest. Radikal rechte Arbeiter bewegen sich in einem gesellschaftlich etablierten Kosmos negativer Klassifikationen. In ihrer sozialen Welt bilden sich Klassen primär aufgrund von Konkurrenz und kollektiver Abwertung, sie entstehen als Wettkampf-Klassen. Arbeiter werten sich selbst auf, indem sie alte und neue, ethnisch geprägte gefährliche Klassen abwerten.

Die völkische Rechte musste diesen Mechanismus nicht erfinden. In Deutschland hat - etwa im Zuge der Arbeitsmarktreformen - eine Mitte-Links-Koalition entsprechende Bewährungsproben durchgesetzt. „Hartz IV“ hat die Schwelle gesellschaftlicher Respektabilität nahe an die aktiven Lohnabhängigenklassen herangerückt (Dörre et al. 2013a). Einmal institutionalisiert, müssen Abwertungen seitens der populistischen Rechten nur noch radikalisiert werden, um die gewünschten symbolischen Effekte zu erzielen. Wer sich, wie unsere Befragten, in dieses System negativer Klassifikationen hineingearbeitet hat und zu seiner Fortschreibung beiträgt, ist nicht nur Opfer (Rommelspacher 1995), wenngleich auch niemals ausschließlich Täter. Bewährungsproben, die stark selektiv wirken, stützen einen Modus negativer Vergesellschaftung. Dieser Modus lässt sich nicht außer Kraft setzen, indem man radikal rechte Kritik an Globalisierung, EU und Migrationsregime durch Attacken gegen ,,antinationale Erziehungsmaßnahmen von oben“ (Streeck 2017, S. 271) zu überbieten versucht. Radikal rechte Arbeiterinnen werden, wie rigide Abschiebepraktiken oder Plädoyers für Flüchtlingsobergrenzen und Asylzentren bewiesen haben, durch solche Eindämmungsstrategien eher noch bestärkt.

Kaum weniger problematisch wirken Analysen, die das Kristallglockenbild übernehmen, um es mit umgekehrten Vorzeichen gegen alle zu wenden, die wohlfahrtsstaatliche Arrangements in der nationalen Arena zumindest ansatzweise verteidigen. Mit universalistischem Gestus tendieren solche Interpretationen dazu, außerhalb der „Komfortzone“ vornehmlich Opfer, in ihrem Inneren hingegen in erster Linie Täter zu verorten. Folgerichtig gilt die Kritik einer ,großen Koalition der Wohlstandbewahrer“, deren Interesse vornehmlich darin bestehe, ihre ,privilegierten Lebensverhältnisse“ gegen die Ausgeschlossenen jenseits der Kristallglocke zu 
verteidigen (Lessenich 2018). Aus dieser Optik heraus wird der völkische Populismus zur Mittelschichtenrevolte stilisiert - eine analytische Verengung, die sich nur aufrecht erhalten lässt, sofern auch noch die Elendesten im Inneren der Kristallglocke zu - subalternen - „Profiteuren“ der herrschenden Weltordnung erklärt werden (Lessenich 2017, S. 64). Von Angehörigen saturierter akademischer Mittelschichten vorgetragen, laufen solche Deutungen in letzter Konsequenz darauf hinaus, dem symbolischen Abwertungsmechanismus demobilisierter Klassengesellschaften zusätzliche Wucht zu verleihen.

Wer die Integrationskraft des rechtspopulistischen Blocks schwächen will, kommt viertens nicht umhin, das Bild von der Kristallglocke insgesamt infrage zu stellen. Homogene Volkskörper sind eine Fiktion - im reichen Norden nicht anders als im armen Süden. Globalisierungsgewinner sind hauptsächlich reiche Eliten, die noch immer überwiegend in den alten Zentren leben. $44 \%$ der Einkommenszuwächse, die zwischen 1988 und 2008 erzielt wurden, entfallen auf die reichsten 5\%, nahezu ein Fünftel auf das reichste $1 \%$ der erwachsenen Weltbevölkerung; die aufstrebenden Mittelklassen in den Schwellenländern verfügen lediglich über 2 bis $4 \%$ der absoluten Zuwächse (Milanovic 2016, 2017). Für die Industriearbeiterschaft, aber auch für das neue Dienstleistungsproletariat der alten Zentren entfällt hingegen zunehmend, was der Ex-Weltbanker Branko Milanovic als „Ortsbonus“ der Vermögensverteilung bezeichnet. Das vermeintliche Privileg, in einem reichen Land geboren zu sein, schützt auch in den reichen Gesellschaften des globalen Nordens schon seit Jahrzehnten nicht mehr vor Armut, Prekarität, Ungleichheit und einem Verlust an Lebenschancen.

Entsprechende Erfahrungen lassen sich gegen die Fiktion homogener Volksgemeinschaften in Stellung bringen. Statt rechten Arbeitern Gerechtigkeits- und Demokratisierungsmotive pauschal abzusprechen, ist es sinnvoll, dem rationalen Kern populistischer Anschauungen größere Aufmerksamkeit zu widmen. Eine kritische Soziologie, die an der Überwindung gesellschaftlicher Klassenvergessenheit zu arbeiten beabsichtigt, findet hier eine Aufgabe. Sie hätte Klassen- und Ausbeutungsverhältnisse wieder öffentlich zu thematisieren und zugleich eine kollektive Diskussion darüber zu ermöglichen, ,welche Themen überhaupt legitim und wichtig sind und daher in Angriff genommen werden sollten“ (Eribon 2016, S. 146). Von einem intakten Resonanzraum, der dies leisten könnte, sind wir gegenwärtig aber meilenweit entfernt. Der letzte Versuch, ,proletarische Öffentlichkeiten“ wissenschaftlich zu fundieren, liegt lange zurück. Oskar Negt und Alexander Kluge (1993) konnten noch davon ausgehen, dass arbeitsorientierte Gegenöffentlichkeiten wenigstens für Verteilungsfragen existierten. In der Gegenwart kann nicht einmal mehr davon die Rede sein. Anders als während der 1980er Jahre lösten selbst Streiks für Arbeitszeitverkürzung mit 1,5 Mio. Beteiligten, die den Einstieg in eine verkürzte Vollzeit von $28 \mathrm{~h}$ und mehr individuelle Zeit für Pflege, Erziehung und Erholung von Schichtarbeit durchgesetzt haben, in der Soziologie wie in der gesamten akademischen Linken nur ein schwaches Echo aus.

Um Mittelschichten-Ressentiments zu überwinden, die solche Auseinandersetzungen als Aktivität privilegierter Arbeiteraristokraten einordnen, müssen auch wissenschaftlich dicke Bretter gebohrt werden. Dabei lässt sich von befragten Arbeiterund Gewerkschafterinnen lernen, die ihre Ablehnung von AfD und Pegida öffentlich 
machen. In ihren eigenen Worten plädieren sie für eine - inklusive - Klassenpolitik, die gemeinsame Interessen ,sagen wir: selbst chinesischer und deutscher Arbeiter“ (Sek1) gegen das dominante Kapital betont. Gewerkschafter, die sich so positionieren, bilden unter den Aktiven noch immer die Mehrheit. Und sie repräsentieren auch die Majorität (potenzieller) Gewerkschaftsmitglieder. Jede gewerkschaftliche Anpassung an die rechtspopulistische Revolte liefe darauf hinaus, die Unterstützung dieser Aktiven aufs Spiel zu setzen. Keiner der Befragten, die den Reichtum der Reichen als zentrale Ursache für die Armut der Armen betrachten (E. O. Wright), käme indes auf die Idee, inklusive Klassenpolitik entwichtige Konflikte, die an den Achsen Geschlecht, Ethnie/Nationalität oder an Naturverhältnissen aufbrechen. Klassenpolitik und gewerkschaftliche Solidarität sind ihrer inneren Logik nach universalistisch. Um Wirkung zu erzielen, müssen sie über Geschlechtergrenzen, Nationalität und ethnische Spaltungen hinweg verbinden. Deshalb sind sie mit völkischen Integrationskonzepten unvereinbar. Umgekehrt sind völkische Ideen, die kulturelle Spaltungen verabsolutieren, ein ideologischer Sprengsatz für solidarische Gewerkschaftspolitik. Wie gezeigt, bietet die betriebliche Arbeitswelt durchaus Erfahrungsräume, in denen Klassenerfahrung in Widerspruch zu völkischem Gedankengut tritt. Daran knüpfen gewerkschaftliche Praktikerinnen an. Selbstbewusst können sie davon ausgehen, dass Bewegungen gegen sexistische und rassistische Diskriminierung ihre größten Erfolge immer dann erzielt haben, wenn auch der ,demokratische Klassenkampf“ (Korpi 1983) zugunsten der Lohnabhängigen einigermaßen erfolgreich war. Die 1968-Revolte entdeckte den Klassenkampf - wenngleich in überhöhter Weise - neu. Zugleich war sie auch eine kulturelle Rebellion für sexuelle Befreiung, Frauenemanzipation, Bürgerrechte und in ihrer Spätwirkung zudem Katalysator für Bewegungen zugunsten ökologischer Nachhaltigkeit (Therborn 2013; Milanovic 2017). Nicht zufällig sind das die Felder, die der völkische Populismus zum Terrain seiner Gegenrevolte erklärt.

In der gesellschaftlichen Auseinandersetzung mit der national-sozialen Gefahr fällt fünftens den Gewerkschaften eine Schlüsselrolle zu. Häufig sind Gewerkschaften die einzigen demokratischen Organisationen, die Arbeiter mit Sympathien für die populistische Rechte überhaupt noch erreichen. Unklar ist jedoch, wie eine erfolgreiche Auseinandersetzung mit dem radikalisierten Populismus aussehen könnte. Dabei sind rechte Betriebsratslisten wie die des ,Zentrum Automobil“, dessen Wurzeln bis in die militante Neonazi-Szene reichen, noch das kleinere Problem. Mit eigenen Listen treten rechte Organisationen in offene Konkurrenz zu gewerkschaftlichen Betriebsräten. Sie sind dann ein äußerer Gegner, der sich aus der Gewerkschaftsperspektive leicht identifizieren lässt. Bei den von uns befragten rechten Arbeiterinnen ist das so nicht der Fall. Ausnahmslos geben sich die Betreffenden als überzeugte Gewerkschafter. Im Betrieb bieten sie keine Angriffsfläche. Außerhalb unserer Schwerpunktregion würden sie sich in gewerkschaftlichen Kontexten als AfD-Sympathisierende gar nicht zu erkennen geben. Deshalb fällt den Gewerkschaften eine Auseinandersetzung mit diesem ,inneren“ Rechtspopulismus schwer.

Erkennbar sind zwei Linien antipopulistischer Politik. Die erste fordert harte Maßnahmen von Unternehmen und Betriebsräten, um autoritäre Charaktere einzuschüchtern (Kern 2018). „Klare Kante“ praktizieren zumeist akademisch gebildete Gewerkschaftssekretäre mit Antifa-Sozialisation, die von ihrem Selbstverständnis 
her gar nicht anders agieren können. Für Anhänger der Gegenposition, die oft einen Arbeiterhintergrund haben, reicht das nicht aus. Beschränkte sich die Organisation auf - juristisch schwer durchsetzbare - Gewerkschaftsausschlüsse, überließe sie mit den ausgeschlossenen Betriebsräten zugleich die repräsentierten Belegschaften der Orientierung durch Pegida und die AfD, lautet das erfahrungsgesättigte Argument. Realitätstaugliche Strategien werden sich zwischen den genannten Polen verorten. Längerfristig muss es deren Hauptanliegen sein, jene kausalen Erklärungsmuster zu verändern, mit deren Hilfe sich das Alltagsbewusstsein Lohnabhängiger Ungleichheit und Unsicherheit verständlich macht. Wichtig ist aus wissenschaftlicher Sicht, dass die Auseinandersetzung offensiv, mit langem Atem und begleitet von einem kontinuierlichen Erfahrungsaustausch geführt wird. Dergleichen mit Hilfe empirischer Forschungen zu unterstützen, ist eine vornehme Aufgabe organischer öffentlicher Soziologie.

Danksagung Der Text beruht auf Forschungen zum Gesellschaftsbild des Prekariats im BMBF-finanzierten Verbund eLabour. Sophie Bose hat einen Teil des Materials für eine Qualifizierungsarbeit ausgewertet (Bose 2017). Die Forschungen waren nur möglich, weil uns Gewerkschafterinnen mutig das Feld öffneten und Befragte ohne Tabus über ihr Verhältnis zu Pegida und der AfD gesprochen haben. Ihnen möchten wir an dieser Stelle bei Wahrung ihrer Anonymität noch einmal ein großes Dankeschön sagen.

Open Access Dieser Artikel wird unter der Creative Commons Namensnennung 4.0 International Lizenz (http://creativecommons.org/licenses/by/4.0/deed.de) veröffentlicht, welche die Nutzung, Vervielfältigung, Bearbeitung, Verbreitung und Wiedergabe in jeglichem Medium und Format erlaubt, sofern Sie den/die ursprünglichen Autor(en) und die Quelle ordnungsgemäß nennen, einen Link zur Creative Commons Lizenz beifügen und angeben, ob Änderungen vorgenommen wurden.

\section{Literatur}

Aulenbacher, B., Burawoy, M., Dörre K., \& Sittel, J. (Hrsg.). (2017). Öffentliche Soziologie. Wissenschaft im Dialog mit der Gesellschaft. Public Sociology - Wissenschaft und gesellschaftsverändernde Praxis. Frankfurt a. M.: Campus.

Bach, S., Thiemann, A., \& Zucco, A. (2018). Looking for the missing rich: Tracing the top tail of the wealth distribution. DIW Discussion Papers, 1717. Berlin: Deutsches Institut für Wirtschaftsforschung.

Balibar, E. (1990). Der „Klassen-Rassismus“. In E. Balibar \& I. Wallerstein, Rasse, Klasse, Nation. Ambivalente Identitäten (S. 247-260). Hamburg: Argument.

Balibar, E. (1993). Die Grenzen der Demokratie. Hamburg: Argument.

Bartels, C. (2018). Einkommensverteilung in Deutschland von 1871 bis 2013: Erneut steigende Polarisierung seit der Wiedervereinigung. DIW-Wochenbericht, 3, 51-62.

Bibouche, S., Held, J., \& Merkle, G. (2009). Rechtspopulismus in der Arbeitswelt. Eine Analyse neuerer Studien. Düsseldorf: Hans-Böckler-Stiftung.

Bieling, H.-J. (2017). Aufstieg des Rechtspopulismus im heutigen Europa. Umrisse einer gesellschaftstheoretischen Erklärung. WSI Mitteilungen, 8, 557-565.

Birsl, U., \& Lösche, P. (2001). (Neo)Populismus in der deutschen Parteienlandschaft, oder: Erosion der politischen Mitte. In D. Loch \& W. Heitmeyer (Hrsg.), Schattenseiten der Globalisierung (S. 346-377). Frankfurt a. M.: Suhrkamp.

Bogner, A., Littig, B., \& Menz, W. (2005). Das Experteninterview. Theorie, Methode, Anwendung. Wiesbaden: VS Verlag für Sozialwissenschaften.

Bohnsack, Ralf (1993). Rekonstruktive Sozialforschung: Einfuihrung in die Methodologie und Praxis qualitativer Forschung. Opladen: Leske + Budrich.

Boltanski, L., \& Chiapello, È. (2003). Der neue Geist des Kapitalismus. Konstanz: UVK.

Bose, S. (2017): Rechtspopulismus und Gewerkschaften. Eine qualitative Untersuchung zu rechten Orientierungen bei aktiven Gewerkschaftsmitgliedern und zum Umgang der Gewerkschaften mit dem Rechtspopulismus. Unveröffentlichtes Manuskript. 
Bourdieu, P. (1988). Die feinen Unterschiede. Kritik der gesellschaftlichen Urteilskraft. 2. Aufl. Frankfurt a. M.: Suhrkamp.

Bourdieu, P. (Hrsg.). (1998). Gegenfeuer. Wortmeldungen im Dienste des Widerstands gegen die neoliberale Invasion. Konstanz: UVK.

Brenke, K., \& Kritikos, A. S. (2017). Wählerstruktur im Wandel. DIW-Wochenbericht, 29, 595-606.

Brinkmann, U., Dörre, K., Röbenack, S., Kraemer, K., \& Speidel, F. (2006). Prekäre Arbeit. Ursachen, Ausmaß, soziale Folgen und subjektive Verarbeitungsformen unsicherer Beschäftigungsverhältnisse. Bonn: Friedrich-Ebert-Stiftung.

Burawoy, M. (2015). Public Sociology. Öffentliche Soziologie gegen Marktfundamentalismus und globale Ungleichheit. Hrsg. v. B. Aulenbacher \& K. Dörre; Nachwort v. H.-J. Urban. Weinheim: Beltz Juventa.

Castel, R. (2000). Die Metamorphosen der sozialen Frage. Eine Chronik der Lohnarbeit. Konstanz: UVK.

Castel, R. (2005). Die Stärkung des Sozialen. Leben im neuen Wohlfahrtsstaat. Hamburg: Hamburger Edition.

Castel, R. (2011). Die Krise der Arbeit. Neue Unsicherheiten und die Zukunft des Individuums. Hamburg: Hamburger Edition.

Decker, F. (2004). Der nеuе Rechtspopulismus. Wiesbaden: VS Verlag für Sozialwissenschaften.

Denzin, N. K. (1989). The research act: A theoretical introduction to sociological methods. New Jersey: Prentice-Hall.

Deppe, F. (1971). Das Bewußtsein der Arbeiter. Studien zur politischen Soziologie des Arbeiterbewußtseins. Köln: Pahl-Rugenstein.

Deppe, F. (2018). Nachwort: Bonapartismus reloaded? In M. Beck \& I. Stützle (Hrsg.), Die neuen Bonapartisten (S. 239-255). Berlin: Dietz.

Destatis (2016). Datenreport 2016. Ein Sozialbericht für die Bundesrepublik Deutschland. https://www. destatis.de/DE/Publikationen/Datenreport/Downloads/Datenreport2016.pdf?_blob=publicationFile. Zugegriffen: März 2018.

DGB (Deutscher Gewerkschaftsbund) (2017). Bundestagswahl 2017. So haben GewerkschafterInnen gewählt. http://www.dgb.de/++co++1aca2e9e-a209-11e7-99c0-525400e5a74a. Zugegriffen: November 2017.

Dörre, K. (2006). Prekarisierung der Arbeitsgesellschaft - Ursache einer rechtspopulistischen Unterströmung? In P. Bathke \& S. Spindler (2006), Neoliberalismus und Rechtsextremismus in Europa. Zusammenhänge - Widersprüche - Gegenstrategien (S. 153-166). Berlin: Dietz.

Dörre, K. (2009). Prekarität im Finanzmarkt-Kapitalismus. In R. Castel \& K. Dörre (Hrsg.), Prekarität, Abstieg, Ausgrenzung. Die soziale Frage am Beginn des 21. Jahrhunderts (S. 35-64). Frankfurt a. M.: Campus.

Dörre, K. (2017). Nach dem schnellen Wachstum: Große Transformation und öffentliche Soziologie. In K. Dörre, B. Aulenbacher, M. Burawoy, \& J. Sittel (Hrsg.), Öffentliche Soziologie - Wissenschaft im Dialog mit der Gesellschaft (S. 33-67). Frankfurt a. M.: Campus.

Dörre, K., Kraemer, K. \& Speidel, F. (2006). Prekäre Beschäftigung und soziale (Des-)Integration. Ursprünge, Konsequenzen und politische Verarbeitungsformen unsicherer Beschäftigung. In FIAB (Forschungsinstitut Arbeit, Bildung, Partizipation) (Hrsg.), Jahrbuch Arbeit, Bildung, Kultur 23/24. Von der Statussicherung zur Eigenverantwortung? Das deutsche Sozialmodell im gesellschaftlichen Umbruch (S. 9-40). Recklinghausen: FIAB.

Dörre, K., Scherschel, K., Booth, M., Haubner, T., Marquardsen, K., \& Schierhorn, K. (2013a). Bewährungsproben für die Unterschicht? Soziale Folgen aktivierender Arbeitsmarktpolitik. Frankfurt a. M.: Campus.

Dörre, K., Happ, A. \& Matuschek, I. (Hrsg.). (2013b). Das Gesellschaftsbild der LohnarbeiterInnen. Soziologische Untersuchungen in ost- und westdeutschen Industriegebieten. Hamburg: VSA.

Dörre, K., Goes, T., Schmalz, S., \& Thiel, M. (2017). Streikrepublik Deutschland? Die Erneuerung der Gewerkschaften in Ost und West. Frankfurt a. M.: Campus.

van Dyk, S., Dowling, E., \& Graefe, S. (2017). Rückkehr des Hauptwiderspruchs? Anmerkungen zur aktuellen Debatte um den Erfolg der Neuen Rechten und das Versagen der ,Identitätspolitik“. PROKLA - Zeitschrift für Kritische Sozialwissenschaft, 47, 411-420.

Engler, W. (2004). Die Ostdeutschen als Avantgarde. Berlin: Aufbau.

Eribon, D. (2016). Rückkehr nach Reims. Berlin: Suhrkamp.

Fiedler, M. (2018). Rechte Kandidaten streben in die Betriebsräte. Der Tagesspiegel, 16.01.2018. https:// www.tagesspiegel.de/themen/agenda/betriebsratswahlen-im-fruehjahr-rechte-kandidaten-strebenin-die-betriebsraete/20849470.html. Zugegriffen: März 2018. 
Fischer, G., Gundert, S., Kawalec, S., Sowa, F., Stegmaier, J., Tesching, K., \& Theuer, S. (2015). Situation atypisch Beschäftigter und Arbeitszeitwünsche von Teilzeitbeschäftigten. Quantitative und qualitative Erhebung sowie begleitende Forschung. IAB-Forschungsprojekt im Auftrag des Bundesministeriums für Arbeit und Soziales. Endbericht 2015. www.doku.iab.de/grauepap/2015/Projektbericht. Zugegriffen: März 2018.

Flick, U. (2008). Qualitative Forschung. Ein Handbuch. Berlin: Rowohlt.

Forschungsgruppe Wahlen e.V. (2017). Wahlanalyse Bundestagswahl. http://www.forschungsgruppe.de/ Wahlen/Wahlanalysen/News1_Bund_170928.pdf. Abgerufen: März 2018.

Fraser, N. (2017). Für eine neue Linke oder: Das Ende des progressiven Neoliberalismus. Blätter für deutsche und internationale Politik, 62(2). https://www.blaetter.de/archiv/jahrgaenge/2017/februar/fuereine-neue-linke-oder-das-ende-des-progressiven-neoliberalismus. Zugegriffen: Dezember 2017.

Fratzscher, M. (2016). Verteilungskampf. Warum Deutschland immer ungleicher wird. München: Carl Hanser.

Friedrich, S. (2016). Falsche Alternativen: Warum breite Bündnisse gegen die AfD keine Perspektive für Linke sind. In H. Kellershohn \& W. Kastrup (Hrsg.), Kulturkampf von rechts. AfD, Pegida und die Neue Rechte (S. 230-234). Münster: Unrast.

Galbraith, J.K. (2016). Wachstum neu denken. Was die Wirtschaft aus den Krisen lernen muss. Zürich: Rotpunkt.

Geiges, L., Marg, S., \& Walter, F. (2015). Pegida. Die schmutzige Seite der Zivilgesellschaft? Bielefeld: Transcript.

Gertz, H. (2017). Superstar. Süddeutsche Zeitung, 30./31.12.2017.

Glaser, B. G., \& Strauss, A. L. (1998). Grounded Theory. Strategien qualitativer Forschung. Bern: Huber.

Gläser, J., \& Laudel, G. (2004). Experteninterviews und qualitative Inhaltsanalyse als Instrumente rekonstruktiver Untersuchungen. Stuttgart: UTB.

Goldthorpe, J. H., Lockwood, D., Bechhofer, F., \& Platt, J. (1967). The affluent worker and the thesis of embourgeoisement: Some preliminary research findings. Sociology, 1, 11-31.

Goodwyn, L. (1978). The populist moment. A short history of the agrarian revolt in America. Oxford: Oxford University Press.

Göttinger Institut für Demokratieforschung (2017). Rechtsextremismus und Fremdenfeindlichkeit in Ostdeutschland. Ursachen und Hintergründe für Rechtsextremismus, Fremdenfeindlichkeit und fremdenfeindlich motivierte Übergriffe in Ostdeutschland sowie die Ballung in einzelnen ostdeutschen Regionen. Abschlussbericht des Forschungsprojekts. Studie im Auftrag der Beauftragten der Bundesregierung für die neuen Bundesländer. http://www.demokratie-goettingen.de/content/uploads/2017/07/ studie-rechtsextremismus-in-ostdeutschland-kurzfassung_offiziell.pdf. Zugriffen: September 2017.

Götze, K. H. (2017). Er fehlt. Heiner Müllers Kritik des Kapitalismus. Das Argument, 323, 288-292.

Gramsci, A. (1991). Gefängnishefte. Kritische Gesamtausgabe. Hamburg: Argument.

Habermas, J. (2016). Für eine demokratische Polarisierung. Blätter für deutsche und internationale Politik, 61(11). https://www.blaetter.de/archiv/jahrgaenge/2016/november/fuer-eine-demokratischepolarisierung. Zugegriffen: Dezember 2017.

Hall, S. (1989). Ausgewählte Schriften. Ideologie, Kultur, Medien, Neue Rechte, Rassismus. Hamburg: Argument.

Hamilton, R. F. (1968). Einkommen und Klassenstruktur. Der Fall der Bundesrepublik. Kölner Zeitschrift für Soziologie und Sozialphilosophie, 20, 250-287.

Hauptmann, A., \& Schmerer, H.-J. (2012). Lohnentwicklung im Verarbeitenden Gewerbe. Wer profitiert vom deutschen Exportboom? IAB Kurzberichte. Aktuelle Analysen aus dem Institut für Arbeitsmarktund Berufsforschung, 20. http://doku.iab.de/kurzber/2012/kb2012.pdf. Zugegriffen: März 2018.

Häusler, A. (2016). Die AfD - eine rechtspopulistische Bewegungspartei? In A. Häusler \& F. Virchow (Hrsg.), Neue soziale Bewegung von rechts? Zukunftsängste. Abstieg der Mitte. Ressentiments (S. 42-51). Hamburg: VSA.

Heitmeyer, W. (2017). Öffentliche Soziologie zu gruppenbezogener Menschenfeindlichkeit. In B. Aulenbacher, M. Burawoy, K. Dörre, \& J. Sittel (Hrsg.), Öffentliche Soziologie. Wissenschaft im Dialog mit der Gesellschaft. Public Sociology - Wissenschaft und gesellschaftsverändernde Praxis (S. 213-227). Frankfurt a. M.: Campus.

Heitmeyer, W. (2018). Autoritärer Nationalradikalismus. Ein neuer Erfolgstypus zwischen konservativem Rechtspopulismus und gewaltförmigem Rechtsextremismus. In K. Becker, K. Dörre \& P. Reif-Spirek (Hrsg.), Arbeiterbewegung von rechts? Frankfurt a. M.: Campus (im Erscheinen).

Hilmer, R., Kohlrausch, B., Müller-Hilmer, R., \& Gagné, J. (2017). Einstellung und soziale Lebenslage. Eine Spurensuche nach Gründen für rechtspopulistische Orientierung, auch unter Gewerkschaftsmitgliedern. Düsseldorf: Hans-Böckler-Stiftung. 
Hochschild, A. (2017a). „Linke müssen erkennen, dass sie sich selbst ins Abseits gestellt haben“. Ruhrbarone, 12.12.2017. https://www.ruhrbarone.de/linke-muessen-erkennen-dass-sie-sich-selbst-insabseits-gestellt-haben/149955. Zugegriffen: März 2018.

Hochschild, A. (2017b). Fremd im eigenen Land. Eine Reise ins Herz der amerikanischen Rechten. Frankfurt a. M.: Campus.

Hörning, K. H. (Hrsg.). (1971). Der ,neue“ Arbeiter. Zum Wandel sozialer Schichtstrukturen. Frankfurt a. M.: Fischer.

IW (Institut der Deutschen Wirtschaft) (2017). Die AfD: eine unterschätzte Partei. Soziale Erwünschtheit als Erklärung für fehlerhafte Prognosen. https://www.iwkoeln.de/fileadmin/publikationen/2017/ 332686/IW-Report_7_2017_Die_AfD_Eine_unterschaetzte_Partei.pdf. Zugegriffen: Februar 2018.

IWF (Internationaler Währungsfond) (2017). World Economic Outlook April 2017. Gaining Momentum? http://www.imf.org/en/publications/weo/issues/2017/04/04/world-economic-outlook-april2017. Zugegriffen: März 2018.

Jongen, M. (2014). Das Märchen vom Gespenst der AfD. Cicero, 22.01.2014. www.cicero.de/innenpolitik/ afd-ein-manifest-fuer-eine-alternative-fuer-europa/56894. Zugegriffen: Februar 2018.

Kadritzke, U. (2017). Mythos „Mitte“. Oder: Die Entsorgung der Klassenfrage. Berlin: Bertz und Fischer.

Kann, M. E. (1983). The new populism and the new marxism. Theory and Society, 12, 365-373.

Kaufmann, J.-C. (1999). Das verstehende Interview. Theorie und Praxis. Konstanz: UVK.

Kelle, U. \& Kluge, S. (2010). Vom Einzelfall zum Typus. Fallvergleich und Fallkontrastierung in der qualitativen Sozialforschung. 2., überarb. Aufl. Wiesbaden: VS Verlag für Sozialwissenschaften.

Kellershohn, H. (2016). Vorbürgerkrieg. In B. Gießelmann, R. Heun, B. Kerst, L. Suermann, \& F. Virchow (Hrsg.), Handwörterbuch rechtsextremer Kampfbegriffe (S. 326-339). Schwalbach am Taunus: Wochenschau.

Kern, P. (2018). AfD schockt die Gewerkschaften. Sozialismus, 45, 48-49.

Kern, H. \& Schumann, M. (1984). Das Ende der Arbeitsteilung? München: C. H. Beck.

Koppetsch, C. (2017). Rechtspopulismus, Etablierte und Außenseiter: emotionale Dynamiken sozialer Deklassierung. In J. Dirk \& O. Nachtwey (Hrsg.), Das Volk gegen die (liberale) Demokratie (S. 208-232). Leviathan Sonderband 32. Baden-Baden: Nomos.

Korpi, W. (1983). The democratic class struggle. London: Routledge \& K. Paul.

Lengfeld, H. (2017). Die „Alternative für Deutschland“: Eine Partei für Modernisierungsgewinner. Kölner Zeitschrift für Soziologie und Sozialpsychologie, 69, 209-232.

Lessenich, S. (2017). Grenzen der Ausbeutung? Wie der globale Norden über die Verhältnisse des Südens lebt. ISW Report, 109, 56-64.

Lessenich, S. (2018). Der Klassenkampf der Mitte. Süddeutsche Zeitung, 02.01.2018.

Loch, D. \& Heitmeyer, W. (Hrsg.). (2001). Schattenseiten der Globalisierung. Rechtsradikalismus, Rechtspopulismus und separatistischer Regionalismus in westlichen Demokratien. Frankfurt a. M.: Suhrkamp.

Mallet, S. (1964). La nouvelle classe ouvrière. Paris: Editions du Seuil.

Mallet, S. (1965). La nouvelle classe ouvrière en France. Cahiers Internationaux de Sociologie, 38, 57-72.

Marx, K. (1960). Der achtzehnte Brumaire des Louis Bonaparte. In K. Marx \& F. Engels, Werke (MEW), Bd. 8 (S. 111-207). Berlin: Dietz.

Mayring, P. (2003). Qualitative Inhaltsanalyse. Weinheim: Beltz.

Mény, Y. \& Sure, Y. (2002). Democracies and the populist challenge. Basingstoke: Palgrave Macmillan.

Merkel, W. (2017). Die populistische Revolte. Kulturpolitische Mitteilungen, 157, 53-56.

Milanovic, B. (2016). Die ungleiche Welt. Migration, das Eine Prozent und die Zukunft der Mittelschicht. Berlin: Suhrkamp.

Milanovic, B. (2017): Haben und Nichthaben. Eine kurze Geschichte der Ungleichheit. Stuttgart: Theiss.

Müller, H. (2017). „Für alle reicht es nicht“. Texte zum Kapitalismus. Berlin: Suhrkamp.

Müller, J.-W. (2016). Was ist Populismus? Ein Essay. Berlin: Suhrkamp.

Nachtwey, O. \& Jörke, D. (2017). Die rechtspopulistische Hydraulik der Sozialdemokratie. In O. Nachtwey \& D. Jörke (Hrsg.), Das Volk gegen die liberale Demokratie (S. 163-186). Leviathan Sonderband 32. Baden-Baden: Nomos

Negt, O., \& Kluge, A. (1993). Geschichte und Eigensinn. Frankfurt a. M.: Suhrkamp.

Patzelt, W. J. (2016). Neun unorthodoxe Thesen zu PEGIDA. In K.-S. Rehberg, F. Kunz \& T. Schlinzig (Hrsg.), PEGIDA. Rechtspopulismus zwischen Fremdenangst und „Wende“-Enttäuschung. Analysen im Überblick (S. 69-83). Bielefeld: Transcript.

Patzelt, W. J., \& Klose, J. (2016). PEGIDA. Warnsignale aus Dresden. Dresden: Thelem.

Paulus, S. (2017). Eine Geschichte der Gegenwart. Zur Sozialen Frage im 21. Jahrhundert. http://www. theoriekritik.ch/?p=2920. Zugegriffen: März 2018. 
Pegida (2015). Programm. Dresdner Thesen. www.pegida.de/programm.html. Zugegriffen: März 2018.

Polanyi, K. (1995). The Great Transformation. Politische und ökonomische Ursprünge von Gesellschaften und Wirtschaftssystemen. 3. Aufl. Frankfurt a. M.: Suhrkamp.

Popitz, H., Bahrdt, H. P., Jüres, E. A., \& Kesting, H. (1957). Das Gesellschaftsbild des Arbeiters. Soziologische Untersuchungen in der Hüttenindustrie. Tübingen: Mohr.

Priester, K. (2012). Rechter und linker Populismus. Annäherung an ein Chamäleon. Frankfurt a. M.: Campus.

Reckwitz, A. (2017). Die Gesellschaft der Singularitäten. Berlin: Suhrkamp.

Rommelspacher, B. (1995). Dominanzkultur. Texte zu Fremdheit und Macht. Berlin: Orlanda.

Sarrazin, T. (2015). Deutschland schafft sich ab. Wie wir unser Land aufs Spiel setzen. München: Deutsche Verlags-Anstalt.

Sauer, D., Stöger U., Bischoff, J., Detje, R., \& Müller, B. (2018). Rechtspopulismus und Gewerkschaften. Eine arbeitsweltliche Spurensuche. Hamburg: VSA.

Schmidt, J. (2015). Arbeiter in der Moderne. Arbeitsbedingungen, Lebenswelten, Organisationen. Frankfurt a. M.: Campus.

Silver, B. J. (2005). Forces of Labor. Arbeiterbewegungen und Globalisierung seit 1870. Hamburg: Assoziation A.

Skidelsky, E. \& Skidelsky, R. (2014). Wie viel ist genug? Vom Wachstumswahn zu einer Ökonomie des guten Lebens. München: Goldmann Verlag.

Sloterdijk, P. (2006). Zorn und Zeit. Politisch-psychologischer Versuch. Frankfurt a. M.: Suhrkamp.

Steil, A. (1984). Die imaginäre Revolte. Untersuchungen zur faschistischen Ideologie und ihrer theoretischen Vorbereitung bei Georges Sorel, Carl Schmitt und Ernst Jünger. Marburg: Arbeiterbewegung und Gesellschaftswissenschaft.

Streeck, W. (2017). Die Wiederkehr der Verdrängten als Anfang vom Ende des neoliberalen Kapitalismus. In H. Geiselberger (Hrsg.), Die große Regression. Eine internationale Debatte über die geistige Situation der Zeit (S. 253-274). Berlin: Suhrkamp.

Taguieff, P.-A. (1991). Die Metamorphosen des Rassismus und die Krise des Antirassismus. In U. Bielefeld (Hrsg.), Das Eigene und das Fremde. Neuer Rassismus in der alten Welt? (S. 221-268). Hamburg: Junius.

Therborn, G. (2013). The killing fields of inequality. London: Polity Press.

Touraine, A. (1964). Une nouvelle classe ouveriére. Sociologie du Travaille, 6(1), 80-84.

Weiß, V. (2017). Die autoritäre Revolte. Die Neue Rechte und der Untergang des Abendlandes. Stuttgart: Klett-Cotta.

Wilkinson, R. \& Pickett, K. (2009). The spirit level. Why more equal societies almost always do better. London: Allen Lane.

Witzel, A. (2000). Das problemzentrierte Interview. Forum Qualitative Sozialforschung/ Forum qualitative Social Research, 1. http://www.qualitative-research.net/index.php/fqs/article/view/1132/2519. Zugegriffen: März 2018.

Wright, E. O. (1985). Classes. London: Verso.

Zeuner, B., Gester, J., Fichter, M., \& Stöss, R. (2007). Gewerkschaften und Rechtsextremismus. Münster: Westfälisches Dampfboot.

Zick, A., \& Küpper, B. (Hrsg.). (2015). Wut, Verachtung, Abwertung. Rechtspopulismus in Deutschland. Bonn: J.H.W. Dietz Nachf.

Klaus Dörre geb. 1957. Dr. phil, Professor für Arbeits-, Industrie- und Wirtschaftssoziologie, Sprecher der DFG-KollegforscherInnengruppe „Landnahme, Beschleunigung, Aktivierung. Dynamik und (De-)Stabilisierung moderner Wachstumsgesellschaften“, Institut für Soziologie, Friedrich-Schiller-Universität Jena; Forschungsschwerpunkte: Kapitalismustheorie/Finanzmarktkapitalismus, flexible und prekäre Beschäftigung, Partizipation in Unternehmen, Arbeitsbeziehungen und Strategic Unionism, Digitalisierung, Rechtspopulismus. Aktuelle Veröffentlichungen: (mit N. Mayer-Ahuja, D. Sauer und V. Wittke) Capitalism and Labor. Towards Critical Perspectives, 2018; Demokratische Klassenpolitik - eine Antwort auf den Rechtspopulismus, in: C. Butterwegge, G. Hentges und B. Lösch (Hrsg.), Auf dem Weg in eine andere Republik? Neoliberalismus, Standortnationalismus und Rechtspopulismus, 2018; (mit K. Becker) Nach dem raschen Wachstum: Doppelkrise und große Transformation, in: L. Schröder und H.-J. Urban (Hrsg.), Gute Arbeit Ausgabe 2018: Ökologie der Arbeit - Impulse für einen nachhaltigen Umbau, 2018. 
Sophie Bose geb. 1990. MA, Wissenschaftliche Mitarbeiterin, DFG-KollegforscherInnengruppe „Landnahme, Beschleunigung, Aktivierung. Dynamik und (De-)Stabilisierung moderner Wachstumsgesellschaften“, Institut für Soziologie, Friedrich-Schiller-Universität Jena. Forschungsschwerpunkte: Arbeitssoziologie, Rechtspopulismus, rechte Orientierungen. Aktuelle Veröffentlichungen: (mit J. Köster u. J. Lütten) Rechte Gewerkschaftsaktive. Gesellschaftsbilder und rechte Einstellungsmuster von aktiven Gewerkschaftsmitgliedern, in: K. Becker, K. Dörre und P. Reif-Spirek (Hrsg.), Arbeiterbewegung von rechts? Ungleichheit - Verteilungskämpfe - Populistische Revolte, Campus, im Erscheinen.

John Lütten geb. 1988. MA, Wissenschaftlicher Mitarbeiter, Institut für Soziologie, Friedrich-SchillerUniversität Jena. Forschungsschwerpunkte: Arbeitssoziologie, Prekarisierung und Prekarität, Lohnabhängigenbewusstseinsforschung. Aktuelle Veröffentlichungen: (mit S. Schmalz und B. Sommer) Prekarität in der chinesischen Hochwachstumsgesellschaft: Eine Fallstudie zur Situation von Wanderarbeitern im Perlflussdelta, in: Berliner Journal für Soziologie, 2017; (mit J. Köster) Die Sekundäranalyse von Gesellschaftsbildern. Vorgehen, Herausforderungen und erste Ergebnisse eines qualitativen arbeitssoziologischen Forschungsprojekts, in: Arbeits- und Industriesoziologische Studien, 2018.

Jakob Köster geb. 1987. MA, Wissenschaftlicher Mitarbeiter, Institut für Soziologie, Friedrich-Schiller-Universität Jena. Forschungsschwerpunkte: Arbeitssoziologie, Prekarisierung und Prekarität. Aktuelle Veröffentlichungen: (mit F. Butollo \& J. Lütten) Von der Informalität zur Prekarität? Die Widersprüche der Re-Regulierung von Arbeit in China, in: Journal für Entwicklungspolitik, 2015; (mit J. Lütten) Die Sekundäranalyse von Gesellschaftsbildern. Vorgehen, Herausforderungen und erste Ergebnisse eines qualitativen arbeitssoziologischen Forschungsprojekts, in: Arbeits- und Industriesoziologische Studien, 2018. 